\title{
Weyl groups, lattices and geometric manifolds
}

\author{
Brent Everitt and Robert B. Howlett *
}

\begin{abstract}
By studying the action of the Weyl group of a simple Lie algebra on its root lattice, we construct torsion free subgroups of small and explicitly determined index in a large infinite class of Coxeter groups. One spin-off is the construction of hyperbolic manifolds of very small volume in up to 8 dimensions.
\end{abstract}

\section{Introduction}

The starting point of this paper is the celebrated theorem that every irreducible representation of the Weyl group of a simple Lie algebra can be realized over $\mathbb{Q}$. The finishing point is the construction of hyperbolic manifolds of very small volume that provide partial solutions to the Siegel problem in low dimensions.

The Weyl groups are examples of Coxeter groups, and it is these, together with the study of their torsion free subgroups, that forms the bridge between the two sentences of the previous paragraph. The realizability over $\mathbb{Q}$ of a linear action $W \rightarrow \mathrm{GL}(V)$ of a Weyl group $W$ is equivalent to the existence of a $W$-invariant lattice $L$ in the ambient space $V$ of the representation. In this paper we use the finite semi-direct product $L \rtimes W$ induced by this action to construct torsion free subgroups inside a large class of Coxeter groups to which the Weyl group $W$ is closely associated. Moreover, the indices of these subgroups are determined explicitly in terms of well known data associated to the Weyl group. As far as we are aware, this is the first such construction of this type.

A feature in recent years of the study of torsion free subgroups of Coxeter groups has been the application to the cnstruction of hyperbolic manifolds. As with any large and seemingly intractable collection of mathematical objects, they are best observed through the eyes of invariants. For hyperbolic manifolds, the most fundamental and important invariant is hyperbolic volume, which "discretises" the situation: the set of possible volumes of hyperbolic manifolds in a fixed dimension form a well-ordered subset of $\mathbb{R}$, discrete even, if the dimension is not three. Moreover, the map vol $: \mathscr{I}_{n} \rightarrow \mathbb{R}$ from the set of isometry classes of finite volume hyperbolic $n$-manifolds is finite to one when $n \neq 2$.

As one of the first general results along these lines was obtained by Carl Ludwig Siegel [26] in 1945, the Siegel (respectively weak Siegel) problem in dimension $n$ is: what is the minimum possible volume obtained by a complete hyperbolic $n$-orbifold (resp. $n$-manifold) without boundary? Our interest is exclusively in the weak problem, which, despite the diminutive (which we will drop from now on), is one with a long history. It can probably be most concisely summarized by saying that its solution in 2-dimensions is classical; in 3-dimensions a solution was announced recently in [14] (at least in the closed orientable case); in 4-dimensions it was first done in [22] (see also [9]) and a solution in 6-dimensions was recently announced in [11].

Many of these solutions have a heavy computational element. Now it turns out that among the Coxeter groups in the large class to which our construction applies, there are a number of

BREnT EveritT: Department of Mathematics, University of York, York YO10 5DD, United Kingdom. e-mail: bje1@york.ac.uk. Robert Howlett: Department of Mathematics, University of Sydney, Sydney NSW 2006, Australia. e-mail: R. Howlett@maths . usyd.edu.au.

* Some of the results of this paper were obtained while the first author was visiting the Department of Mathematics, University of Sydney and the Institute for Geometry and its Applications, University of Adelaide. He is grateful for their hospitality and financial assistance. The research done at the University of Sydney was supported by a grant from the Australian Research Council. 
geometric examples, ie: Coxeter groups generated by reflections in the faces of a Coxeter polytope in hyperbolic space. When specialized to these cases, our results yield manifolds that are of very small volume, often only twice the volume of a possible solution to the Siegel problem. They also have the virtue of being constructed algebraically rather than computationally.

The paper has been written so as to include in its audience workers in the field of geometric manifolds who may be less familiar with the technical details of Weyl groups and Coxeter groups. The first section is therefore a summary of the basic definitions and some of the results we will need later. Sections 2, 3and 4, while the technical heart of the paper, can be glossed over on the first reading by those impatient to get to the geometrical consequences in $\$ 5$, The main results are Theorems 7,10 .

\section{Preliminaries on reflection groups}

General references for this section are [2, 17, 19,30]; the terminology below mostly follows [17].

\subsection{Coxeter groups and reflection groups}

Let $W$ be a group and $S \subset W$ finite. The pair $(W, S)$ is called a Coxeter group if $W$ admits a presentation with generators the $s \in S$, and relations,

$$
(s t)^{m_{s t}}=1
$$

where $m_{s t}=m_{t s} \in \mathbb{Z}^{+} \cup\{\infty\}$ and $m_{s t}=1$ if and only if $s=t$. Associated to any Coxeter group is its symbol $\Gamma$, with nodes the $s \in S$, and where nodes $s$ and $t$ are joined by an edge labeled $m_{s t}$ if $m_{s t} \geq 4$ and an unlabeled edge if $m_{s t}=3$ (and so nodes $s$ and $t$ not connected correspond to $m_{s t}=2$ ). Conversely, any symbol $\Gamma$ with edges so labeled gives rise to Coxeter group $W(\Gamma)$, with generators the nodes of the symbol and relations (1) with $m_{s t}$ the label of the edge connecting nodes $s$ and $t$.

If $T \subset S$ then the subgroup generated by the $s \in T$ is also a Coxeter group with symbol $\Delta \subset \Gamma$ obtained by removing the nodes not in $T$ and any edges incident with them. Write $W_{T}$ or $W(\Delta)$ for this, a visible subgroup of $W(\Gamma)$ (with parabolic, special parabolic and visual being alternative terms found in the literature). The Coxeter group $W(\Gamma)$ is irreducible when $\Gamma$ is connected, and if $\Gamma$ has connected components $\Gamma_{i} \subset \Gamma$, then the visible $W\left(\Gamma_{i}\right)$ are called the irreducible components of $W(\Gamma)$, with $W(\Gamma)$ the direct product of the $W\left(\Gamma_{i}\right)$.

A Coxeter group $W(\Gamma)$ can be realized as a reflection group via the reflectional (or geometric, or Tits) representation: let $V$ be the real vector space with basis $\left\{v_{s} \mid s \in S\right\}$ and symmetric bilinear form defined by,

$$
B\left(v_{s}, v_{t}\right)=-\cos \frac{\pi}{m_{s t}} .
$$

When $m_{s t}=\infty$, set the product to be some real number $c_{s t}$ with $c_{s t} \leq-1$. For $u \in V$, define $\sigma_{u}: V \rightarrow V$ by $\sigma_{u}(v)=v-2 B(v, u) u$, and then the map $s \mapsto \sigma_{s}:=\sigma_{v_{s}}$ extends to a faithful representation $\sigma: W(\Gamma) \rightarrow \mathrm{GL}(V)$, irreducible when $W(\Gamma)$ is irreducible and $B$ non-degenerate. We will abbreviate $\sigma(g)(v)$ to $g(v)$.

The two most important classes of Coxeter groups for us will be the finite and hyperbolic: $W(\Gamma)$ is finite if and only if the form $B$ is positive definite, in which case $V$ is a Euclidean space with $W(\Gamma)$-invariant inner product $(v, u):=B(v, u)$.

A hyperbolic Coxeter polytope is an intersection $P=\bigcap_{s \in S} H_{s}^{-}$, of closed half spaces $H_{s}^{-}$in $n$-dimensional hyperbolic space $\mathbb{H}^{n}$, bounded by hyperplanes $H_{s}$, any two of which are disjoint or subtend a dihedral angle of $\pi / m_{s t}$. The group generated by reflections in the faces of $P$ is then a Coxeter group, and an abstract $W(\Gamma)$ is $n$-dimensional hyperbolic in this sense if there exist $c_{s t}$ 's such that the form $B$ above has signature $(1, n)$. In this case $c_{s t}=-\cosh \eta_{s t}$, for $\eta_{s t}$ the distance between non-intersecting $H_{s}$ and $H_{t}$. 


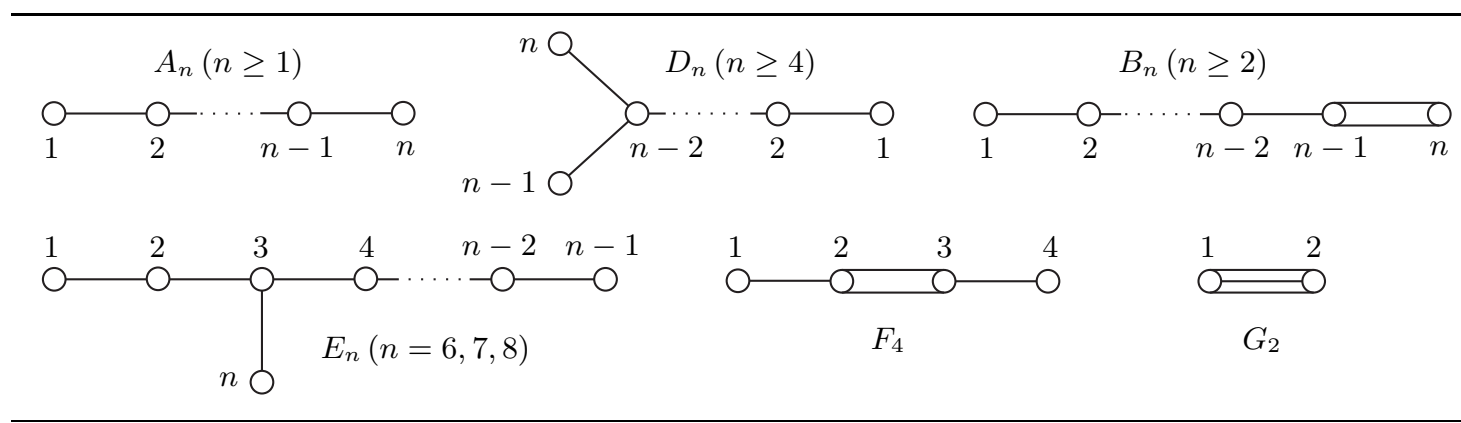

Table 1. The irreducible Weyl groups

\subsection{Crystallographic groups and Weyl groups}

A Coxeter group $W(\Gamma)$ is crystallographic if in the reflectional representation $W(\Gamma) \rightarrow \mathrm{GL}(V)$ there is a $W(\Gamma)$-invariant lattice $L \subset V$ (one often sees in the literature a somewhat larger class of Coxeter groups referred to as crystallographic, namely the Weyl groups of Kac-Moody Lie algebras). A finite crystallographic Coxeter group is a Weyl group, and if $(u, v)$ is the inner product on $V$ from $₫ 1.1$ let

$$
\langle u, v\rangle:=\frac{2(u, v)}{(u, u)} .
$$

The symbols for the irreducible Weyl groups are given in Table 1, using the alternative symbol convention where nodes $v_{s}$ and $v_{t}$ are joined by $\left\langle v_{s}, v_{t}\right\rangle\left\langle v_{t}, v_{s}\right\rangle$ unlabelled edges. We will tend to use this version when some additional labeling of the nodes might otherwise lead to an overly cluttered picture 1 We have fixed a numbering of $S$ in the table, and throughout the paper we will write $v_{i}:=v_{s_{i}}$ for the basis of the ambient space $V$ of the reflectional representation.

We use the Killing-Cartan notation from the classification of simple Lie algebras to denote the symbols for the irreducible Weyl groups. There are three infinite classical families, $A_{n}(n \geq$ $1), B_{n}(n \geq 2)$ and $D_{n}(n \geq 4)$, together with five exceptional groups $G_{2}, F_{4}, E_{6}, E_{7}$ and $E_{8}$. The subscripts give the rank of the group, which is just the number of nodes of the symbol.

There are a number of $W(\Gamma)$-invariant lattices in $V$, with the most fundamental being the root lattice, $L(\Gamma)=\mathbb{Z}$-span $\left\{x_{s} \mid s \in S\right\}$, where $x_{s}=v_{s}$ in types $A, D$ and $E$. Otherwise, for $L\left(B_{n}\right)$, let $x_{i}=v_{i}(i<n)$ and $x_{n}=\sqrt{2} v_{n}$; in $L\left(F_{4}\right)$ we have $x_{1}=v_{1}, x_{2}=v_{2}, x_{3}=$ $\sqrt{2} v_{3}, x_{4}=\sqrt{2} v_{4}$ and $L\left(G_{2}\right)=\mathbb{Z}$-span $\left\{x_{1}=v_{1}, x_{2}=\sqrt{3} v_{2}\right\}$. The $W(\Gamma)$-action on the root lattice realizes the reflectional representation over $\mathbb{Q}$, and remarkably, any irreducible representation of a Weyl group can be so realized (see [29]).

There is another interesting $W(\Gamma)$-invariant lattice in $V$ : the weight lattice $\widehat{L}=\widehat{L}(\Gamma)$ is given by $\widehat{L}=\{v \in V \mid\langle u, v\rangle \in \mathbb{Z}$ for all $u \in L\}$. One can also define $\widehat{L}$ indirectly as the dual of the coroot lattice. The weight lattice has basis the simple weights $\omega_{s}$ defined by $\left\langle v_{s}, \omega_{s}\right\rangle=1$ and $\left\langle v_{t}, \omega_{s}\right\rangle=0$ for $t \neq s$. In particular $\widehat{L}$ is a $\mathbb{Z}$-lattice of the same rank as $L$ and with $L \subset \widehat{L}$. Thus the root lattice is a subgroup of finite index in the weight lattice, with this index called the index of connection (see Table 2).

The classical Weyl groups have alternative descriptions as groups of permutations of an orthonormal basis for $V$ (or a space closely related to it). For type $A_{n}$ and basis $\left\{v_{1}, \ldots, v_{n}\right\}$, let $\widehat{V}$ be an $(n+1)$-dimensional Euclidean space with orthonormal basis $\left\{u_{1}, \ldots, u_{n+1}\right\}$, and embed $V \hookrightarrow \widehat{V}$ via,

$$
v_{i}=\frac{1}{\sqrt{2}}\left(u_{i}-u_{i+1}\right) \text { for } 1 \leq i \leq n .
$$

The $W\left(A_{n}\right)$-action on $V$ extends to $\widehat{V}$ with the $\left\{u_{i}\right\}$ a $W\left(A_{n}\right)$-invariant subset and the map $s_{i} \mapsto\left(u_{i}, u_{i+1}\right)$ inducing an isomorphism $W\left(A_{n}\right) \rightarrow \mathfrak{S}\left(u_{1}, \ldots, u_{n+1}\right) \cong \mathfrak{S}_{n+1}$, the symmetric group of degree $n+1$.

\footnotetext{
1 The Weyl groups constitute almost all the finite Coxeter groups, with the only omissions being the dihedral groups and the symmetry groups of the 3-dimensional dodecahedron/icosahedron and the 4-dimensional 120/600-cell.
} 


\begin{tabular}{cccccc}
\hline$\Gamma$ & basis $\left\{x_{i}\right\}$ for $L$ & index of connection & $(-1)$-type & Coxeter number & exponents \\
\hline$A_{n}$ & $\left\{v_{i}\right\}$ & $n+1$ & $n=1$ & $n+1$ & $1,2, \ldots, n$ \\
$B_{n}$ & $\left\{v_{i}(i<n), \sqrt{2} v_{n}\right\}$ & 2 & $\checkmark$ & $2 n$ & $1,3, \ldots, 2 n-1$ \\
$D_{n}$ & $\left\{v_{i}\right\}$ & 4 & $n$ even & $2(n-1)$ & $1,3, \ldots, 2 n-3, n-1$ \\
$G_{2}$ & $\left\{v_{1}, \sqrt{3} v_{2}\right\}$ & 1 & $\checkmark$ & 6 & 1,5 \\
$F_{4}$ & $\left\{v_{1}, v_{2}, \sqrt{2} v_{3}, \sqrt{2} v_{4}\right\}$ & 1 & $\checkmark$ & 12 & $1,5,7,11$ \\
$E_{6}$ & $\left\{v_{i}\right\}$ & 3 & $\times$ & 12 & $1,4,5,7,8,11$ \\
$E_{7}$ & $\left\{v_{i}\right\}$ & 2 & $\checkmark$ & 18 & $1,5,7,9,11,13,17$ \\
$E_{8}$ & $\left\{v_{i}\right\}$ & 1 & $\checkmark$ & 30 & $1,7,11,13,17,19,23,29$ \\
\hline
\end{tabular}

Table 2. The Weyl group data discussed in $\$ 1.2$

For types $B$ and $D$, let $\mathfrak{S}^{ \pm}(X)$ be the group of signed permutations of the set $X$, ie: $\mathfrak{S}^{ \pm}(X)=\{\sigma \in \mathfrak{S}(X \cup-X) \mid \sigma(-x)=-(\sigma x)\}$. The vectors $u_{1}, \ldots, u_{n} \in V$ defined by

$$
v_{i}=\frac{1}{\sqrt{2}}\left(u_{i}-u_{i+1}\right) \text { for } i<n, \text { and } v_{n}=u_{n},
$$

form an orthonormal basis for $V$ and the map $s_{i} \mapsto\left(u_{i}, u_{i+1}\right)\left(-u_{i},-u_{i+1}\right), 1 \leq i<n$ and $s_{n} \mapsto\left(u_{n},-u_{n}\right)$ induces an isomorphism $W\left(B_{n}\right) \rightarrow \mathfrak{S}^{ \pm}\left(u_{1}, \ldots, u_{n}\right)$. Taking instead $v_{n}=$ $\frac{1}{\sqrt{2}}\left(u_{n-1}+u_{n}\right)$ and $s_{n} \mapsto\left(u_{n-1},-u_{n}\right)\left(u_{n},-u_{n-1}\right)$, induces an isomorphism $W\left(D_{n}\right) \hookrightarrow$ $\mathfrak{S}^{ \pm}\left(u_{1}, \ldots, u_{n}\right)$ onto a subgroup of index two, the even signed permutations $\mathfrak{S}_{\circ}^{ \pm}\left(u_{1}, \ldots, u_{n}\right)$.

Every Coxeter group has a length function $\ell: W(\Gamma) \rightarrow \mathbb{Z}^{\geq 0}$, where $\ell(g)$ is the smallest $r$ for which an expression $g=s_{1} s_{2} \ldots s_{r}$ exists, with the $s_{i} \in S$. In particular if $W(\Gamma)$ is finite, then there is a unique $w_{\Gamma}$ with maximal length, which, significantly for us, turns out to be an involution. If the $\Gamma_{i}$ are the connected components of $\Gamma$ and $\ell_{i}$ the length functions on the $W\left(\Gamma_{i}\right)$, then $\ell=\sum \ell_{i}$ is the length function on $W(\Gamma)$.

A Coxeter group $W(\Gamma)$ is of $(-1)$-type if in the reflectional representation there is an element $g \in W$ acting on $V$ as the antipodal map, ie: $g(v)=-v$ for all $v \in V$ (and then $g$ is the element $w_{\Gamma}$ of longest length $\sqrt{2}$. An irreducible group has $(-1)$-type precisely when it has non-trivial center; every $W$ of $(-1)$-type is finite; and $W$ is of (-1)-type if and only if its irreducible components are of $(-1)$ type [2, Chapter V, $\S 4$, Exercises 2(b) and 3(c)] or [24, 1.9].

A product involving all the elements of $S$ precisely once is called a Coxeter element, and if the symbol $\Gamma$ is a tree (in particular, if we have a Weyl group) then any two Coxeter elements are conjugate. In particular, the Coxeter elements all have the same order, the Coxeter number $h$. In the reflectional representation, the Coxeter elements have eigenvalues, $\zeta^{m_{1}}, \zeta^{m_{2}}, \ldots, \zeta^{m_{n}}$, with $\zeta$ a primitive $h$-th root of unity, and $0 \leq m_{1} \leq m_{2} \leq \cdots \leq m_{n}<h$ the exponents of $W(\Gamma)$. Every integer $1 \leq m<h$ relatively prime to $h$ is an exponent (although there may be others), and the order of a Weyl group is the product of the $m_{i}+1$.

\subsection{Torsion in Coxeter groups}

In $\S \S 3$, we will want to show that certain subgroups of Coxeter groups are torsion free, ie: contain no non-trivial elements of finite order, and the arguments will require a detailed knowledge of the location in a Coxeter group of the torsion. The first key result gives a rough description:

Theorem 1 ([2, V.4.2], [3, Proposition 1.3]). Any element of finite order in a Coxeter group $W(\Gamma)$ is conjugate to an element of a finite visible subgroup $W(\Delta)$ for $\Delta \subset \Gamma$.

See also [9. Theorem 1] for a geometrical proof. In particular, if $f: W(\Gamma) \rightarrow G$ is a homomorphism, then ker $f$ is torsion free precisely when $f$ is faithful on the finite visible $W(\Delta)$ for $\Delta \subset \Gamma$. Indeed, if $\Delta_{i} \subset \Gamma$ are disjoint symbols with $f W\left(\Delta_{1}\right) \cap f W\left(\Delta_{2}\right)=\{1\}$, then $f$

\footnotetext{
${ }^{2}$ Geometrically, every Weyl group is the symmetry group of a solid polytope, indeed a regular polytope in types $A, B$ and $F$. Then $W$ has $(-1)$-type when there is polytope whose vertices occur in antipodal pairs. These polytopes are all familiar except possibly in type $E$ (see [7]).
} 
is faithful on $W\left(\Delta_{1} \coprod \Delta_{2}\right)$ if and only if $f$ is faithful on the $W\left(\Delta_{i}\right)$, so it is often possible to reduce consideration to the irreducible finite visibles.

Clearly a group is torsion free if it is free of $p$-torsion (elements of order $p$ ) for all primes $p$. The following is then typical of the results we will need, and follows from [4, §7] or [9, Theorem $5]$.

Lemma 1. Let $g \in W\left(B_{n}\right)$ be an element of p-torsion for $p>2$ a prime. Then $g$ is conjugate to an element of the visible $W\left(A_{n-1}\right)=\left\langle s_{1} \ldots, s_{n-1}\right\rangle$.

Because of the lemma, it will turn out that most of our efforts will be focused on the 2-torsion, for which there is a more precise classification due to Richardson [24] which we now summarize (see also [8, 16]). First, if $W(\Psi)$ is an irreducible Weyl group, define a permutation $\pi_{\Psi}$ of the nodes of $\Psi$ as follows: if $\Psi$ is of (-1)-type then $\pi_{\Psi}$ is the identity permutation, otherwise, it is the unique symmetry of order 2 of the diagram. Now let $\Gamma$ be a symbol for any $(W, S)$ and $\Delta \subset \Gamma$ a subsymbol of $(-1)$-type with nodes $T \subset S$; for $s \in S$, let $\Delta(s)$ be the connected component of $T \cup\{s\}$ containing $s$; let $\mathcal{A}(\Delta)$ be those nodes $s \in S \backslash T$ such that $\Delta(s)$ is not a symbol of (-1)-type.

Two subsymbols $\Delta, \Delta^{\prime} \subset \Gamma$ are connected by an elementary $W(\Gamma)$-equivalence, written $\Delta \vdash_{s} \Delta^{\prime}$, if $\Delta^{\prime}$ has nodes $T \cup\{s\} \backslash\left\{s^{\prime}\right\}$, where $s \in \mathcal{A}(\Delta)$ and $s^{\prime}=\pi_{\Delta(s)}(s)$. The two symbols are $W(\Gamma)$-equivalent if there is a sequence $\Delta=\Delta_{0} \vdash_{s_{1}} \Delta_{1} \vdash_{s_{2}} \cdots \vdash_{s_{k}} \Delta_{k}=\Delta^{\prime}$ of elementary equivalences between them.

Theorem 2 ([24, Theorem A]). The map $\Delta \mapsto w_{\Delta}$ induces a bijection from the set $\mathcal{J}_{\Gamma}$ of $W(\Gamma)$ equivalence classes of subsymbols $\Delta \subset \Gamma$ of (-1)-type to the set of conjugacy classes of involutions in the Coxeter group $W(\Gamma)$.

For example, if $\Gamma=A_{n}$ ( $n$ even) with subsymbols $\Delta, \Delta^{\prime}$ the nodes $\left\{s_{i} \mid i\right.$ odd $\}$ and $\left\{s_{i} \mid\right.$ $i$ even $\}$ respectively, then there is a series of elementary $W(\Gamma)$-equivalences that successively, working from right to left, move the nodes of $\Delta$ one place to the right, and so realizing a $W(\Gamma)$ equivalence between $\Delta$ and $\Delta^{\prime}$.

As a corollary, if $W(\Gamma)$ is an irreducible Weyl group, then there is a unique class in $\mathcal{J}_{\Gamma}$ containing symbols of maximal rank. Indeed, as an inspection of Table 1 shows, this class contains a single symbol, except when $\Gamma=A_{n}$ ( $n$ even), when it contains the two symbols $\Delta, \Delta^{\prime}$ of the previous paragraph.

Proposition 1. Let $W(\Gamma)$ be an irreducible Weyl group with Coxeter number heven, $\xi \in W(\Gamma)$ a Coxeter element, and $\Delta \in \mathcal{J}_{\Gamma}$ of maximal rank. Then $\xi^{h / 2}$ is conjugate to $w_{\Delta}$.

Proof. By checking separately the cases, $\Gamma$ of $(-1)$-type, $\Gamma=A_{n}(n$ odd $), \Gamma=D_{n}(n$ odd $)$, and $\Gamma=E_{6}$, one can show, using the exponents in Table 2, that the eigenvalues of $\xi^{h / 2}$ are $-1, \ldots,-1,1 \ldots, 1$, with the rank of $W(\Delta)$ number of -1 's.

\section{Technical results on root lattices}

This section studies in some detail the action of a Weyl group on certain vector spaces over the field $\mathbb{F}_{2}$ of order two. The reader who is more interested in the broad flow of the paper than this somewhat intricate combinatorics (one imagines this describes most readers) can safely skip the details: the key ideas are the sublattices $\Lambda_{s} \subset L$ defined after Lemma 2 , the notions of admissibility and special admissibility after Proposition 5, and Theorems 3.5. These all play an essential role in $\$ 3$, while Theorem 6 and Proposition 6 are used in $\$ 4$

Lemma 2. Let $W(\Psi)$ be an irreducible Weyl group of rank $n$, and $W(\Psi) \rightarrow G L(V)$ the reflectional representation. Then for any node $s \in \Psi$, there is $a u_{s}$ in the root lattice $L=\mathbb{Z}$-span $\left\{x_{i}\right\}$ such that $u_{s} \in\left\langle x_{1} \ldots, \widehat{x}_{s}, \ldots, x_{n}\right\rangle^{\perp}$. 


\section{Proof.}

1. slick proof: let $u_{s}=|\widehat{L} / L| \omega_{s}$, with $\omega_{s}$ the simple weight corresponding to $s$ and $|\widehat{L} / L|$ the index of connection.

2. simple-minded proof (but useful for later calculations): Depict $u_{s}=\sum_{t \in S} \alpha_{t} x_{t}$ using the symbol $\Psi$ by labeling the node $t$ by $\alpha_{t}$ and colouring black the distinguished node corresponding to $s$. Letting $\lambda_{s t}=\left\langle v_{s}, v_{t}\right\rangle\left\langle v_{s}, v_{t}\right\rangle$, we have that $u_{s}$ has the desired properties if and only if for every $t \in S \backslash\{s\}$,

$$
2 \alpha_{t}=\sum \lambda_{s^{\prime} t} \alpha_{s^{\prime}}
$$

where the sum is over all $s^{\prime} \in S$ with $m_{s^{\prime} t}>2$ (ie: all $s^{\prime}$ connected by an edge to $t$ ), except if $t$ is the node in $B_{n}, F_{4}$ or $G_{2}$ with an $m_{s t}=4$ or 6 , and $x_{t}=\sqrt{2}$ or $\sqrt{3}$ times the length of $v_{t}$. In this case we require

$$
2 \alpha_{t}=\sum \alpha_{s^{\prime}}
$$

instead. Thus it suffices to show that for any connected $\Psi$ and distinguished black node $s$, the vertices can be $\mathbb{Z}$-labeled satisfying conditions (2) and (3). We can almost always reduce to the case where $s$ lies at the end of $\Psi$, for suppose $\Psi_{1}, \Psi_{2}$ have distinguished black nodes at the end, labeled $\alpha_{i}$ and satisfying (2) and (3) above. Multiply the labels of $\Psi_{i}$ by $\alpha_{j} / \operatorname{gcd}\left(\alpha_{1}, \alpha_{2}\right)$ and fuse the black nodes:
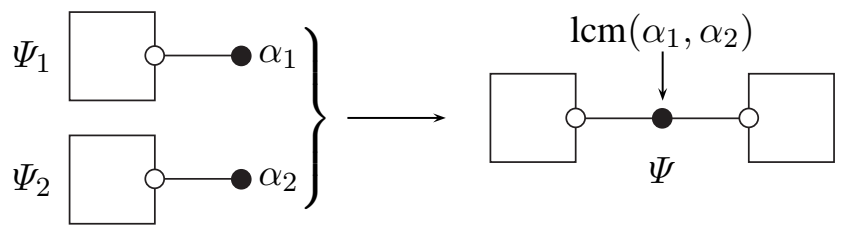

The new symbol $\Psi$ has a $\mathbb{Z}$-labeling satisfying (2) and (3) as long as in passing from the unfused to the fused symbols, no node moves from having to satisfy (2) to having to satisfy (3) or vice-versa. But the only time this happens is for the single pair $\left(F_{4}, s_{2}\right)$, which we deal with separately. Thus, we just need to exhibit labelings in the other cases, where $s$ is at the end of $\Psi$. In types $A$ and $G$ they are,
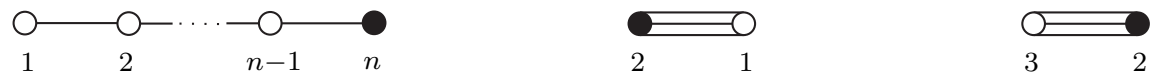

The asymmetry in $G_{2}$, despite the symmetry of the symbol, is due to the asymmetry of the basis $\left\{x_{1}, x_{2}\right\}$ for the $G_{2}$-root lattice. In type $F$, we have,
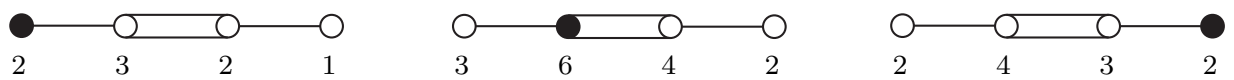

In type $D$ we have,
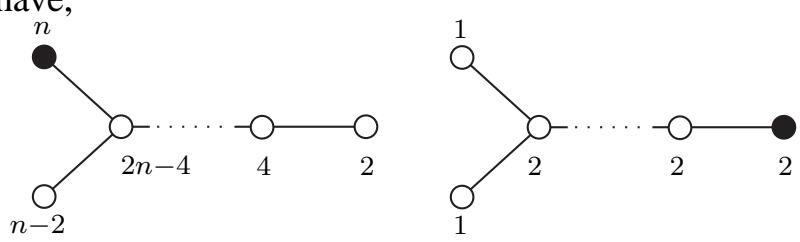

removing the factor of 2 on the left when $n$ is even; in type $B$ we have,
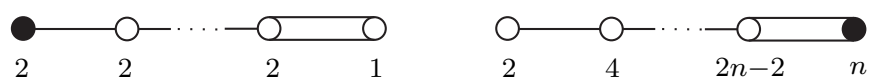

again removing factors of 2 , and finally in type $E$ :

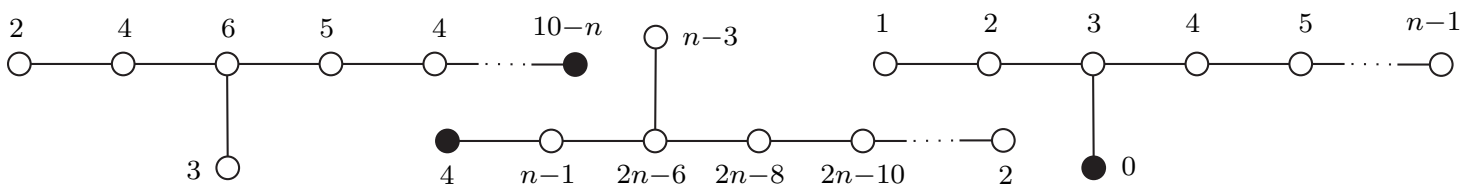


In the middle situation for $E_{7}$, remove the factor of 2 that appears in each label.

Now for the key player in this section. Let $W(\Psi)$ be an irreducible Weyl group of rank $n$, $s \in \Psi$ and $u_{s} \in L$ the vector of Lemma 2. Let $\Omega_{s} \subset L$ be the orbit under $W(\Psi)$ of $u_{s}$ and $\Lambda_{s} \subset L$ the sublattice spanned by $\Omega_{s}$. Thus $U_{s}=\Lambda_{s} \otimes \mathbb{R}$ is a $W(\Psi)$-invariant subspace of $V$, and as the reflectional representation is irreducible we have $U_{s}=V$, and so $\Lambda_{s}$ is a free $\mathbb{Z}$-module, of the same rank $n$ as $L$.

Consider $L / 2 L:=L / 2$, an $n$-dimensional vector space over $\mathbb{F}_{2}$, the subspace $\Lambda_{s} / 2 \subset L / 2$, and write $\bar{v}$ for the image of $v \in L$ via the quotient map $L \rightarrow L / 2$. When making statements about $\bar{u}_{s}$ in the remainder of the paper, it is important to use the explicit $u_{s}$ given in the proof of Lemma 2 (particularly with factors of 2 removed!). As $2 L$ is a $W(\Psi)$-invariant submodule, the $W(\Psi)$-actions on $L$ and $\Lambda_{s}$ induce actions on $L / 2$ and $\Lambda_{s} / 2$.

Let $s, t \in \Psi$, and since the Weyl groups all have symbols that are trees, there is a unique path containing a minimal number of edges connecting $s$ to $t$, with nodes $s=s_{1}, \ldots, s_{k}=t$ say. Let,

$$
X_{t}^{s}=\left\{\bar{u}_{s}, s_{1}\left(\bar{u}_{s}\right), s_{2} s_{1}\left(\bar{u}_{s}\right), \ldots, s_{k} \cdots s_{2} s_{1}\left(\bar{u}_{s}\right)\right\},
$$

a collection of (at most) $k+1$ vectors in $\Lambda_{s} / 2$. Observe that for $t^{\prime} \in \Psi$, the sets $X_{t}^{s}$ and $X_{t^{\prime}}^{s}$ intersect in $\ell+1$ vectors when the paths connecting $s$ to $t$ and $t^{\prime}$ have in common an initial path containing $\ell$ edges, so in particular, $X_{t}^{s} \cap X_{t^{\prime}}^{s}$ always contains at least two vectors.

For $s \in \Psi$, call a set of nodes $T \subset \Psi$ independent for $s$ when $\bigcup_{t \in T} X_{t}^{s}$ contains $m+1$ linearly independent vectors in $\Lambda_{s} / 2$, where $m$ is the number of nodes contained in the union of all the minimal paths connecting $s$ to the $t \in T$.

Finally, the independence data for $W(\Psi)$ is a labeling of the nodes of $\Psi$ by strings of the form,

$$
t_{1}, \ldots, t_{k}, \overline{t_{11}, \ldots, t_{1 n_{1}}}, \ldots, \overline{t_{\ell 1}, \ldots, t_{\ell_{\ell}}} \text {, with the } t \text { 's in } \Psi
$$

such that if $T$ is a union of any subset of the $t_{1}, \ldots, t_{k}$ together with the $t$ 's in exactly one of the $\overline{t_{i 1}, \ldots, t_{i n_{i}}}$, then $T$ is independent for the node $s$ having this label. As always, the node labels follow the conventions in Table 1 , and if a node $s$ is labeled by 0 , this indicates that $\left\{\bar{u}_{s}\right\}$ is non-zero, but the vectors in $X_{t}^{s}$ are not independent for any $t$ (including $t=s$ ).

For example, in Proposition 4 an $s$ that is $\ell \equiv 2 \bmod 4$ nodes from the righthand end of $\Psi=D_{n}$ has label $2, \overline{1, n}, \overline{1, n-1}, \overline{n, n-1}$, indicating that, say

$$
\begin{aligned}
X_{2}^{s} \cup X_{1}^{s} \cup X_{n-1}^{s}=X_{1}^{s} \cup X_{n-1}^{s}=\{ & \left\{\bar{u}_{s}, s_{\ell}\left(\bar{u}_{s}\right), s_{\ell-1} s_{\ell}\left(\bar{u}_{s}\right), \ldots, s_{1} \cdots s_{\ell}\left(\bar{u}_{s}\right),\right. \\
& \left.s_{\ell+1} s_{\ell}\left(\bar{u}_{s}\right) \ldots, s_{n-1} \cdots s_{\ell}\left(\bar{u}_{s}\right)\right\},
\end{aligned}
$$

consists of $n$ independent vectors in $\Lambda_{s} / 2$. The barred $t$ 's are meant to stop us from getting too carried away: $X_{1}^{s} \cup X_{n-1}^{s} \cup X_{n}^{s}$ for instance contains too many (ie: more than $n$ ) distinct vectors to be independent.

Proposition 2. The independence data for $\Psi=A_{n}$ is,

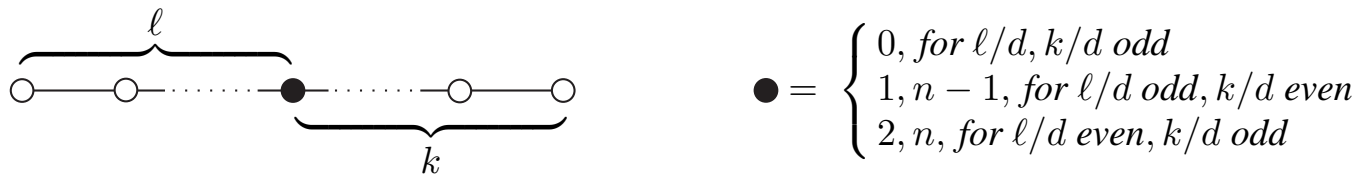

where $d=\operatorname{gcd}(\ell, k)$.

Proof. For the node $s$ indicated the vector $u_{s} \in L$ is given by,

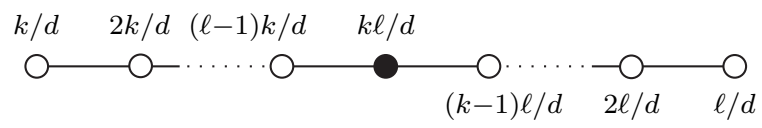

where at least one of $\ell / d$ and $k / d$ must be odd. Thus $\bar{u}_{s}=10 \cdots 101 \cdots 01$ or $10 \cdots 010 \cdots 01$ in the first case listed at the right in the Lemma, and $00 \cdots 001 \cdots 01$ or $10 \cdots 100 \cdots 00$ in the other two. It's a straight out calculation from here on in. 
Proposition 3. The independence data for $\Psi=B_{n}$ is,

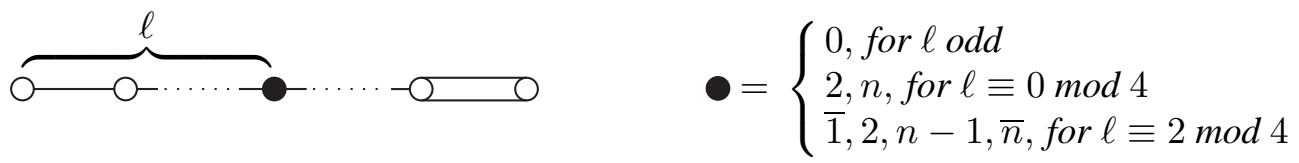

and the labeling of the right-most node is 0 for $n$ odd, and $n$ for $n$ even.

The proof is completely analogous to Proposition 2, as is the proof of:

Proposition 4. The independence data for $\Psi=D_{n}$ is,

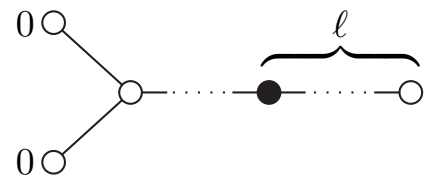

$$
\boldsymbol{\bullet}=\left\{\begin{array}{l}
0, \text { for } \ell \text { odd } \\
2, n-1, n, \text { for } \ell \equiv 0 \bmod 4 \\
2, \overline{1, n}, \overline{1, n-1}, \overline{n, n-1}, \text { for } \ell \equiv 2 \bmod 4
\end{array}\right.
$$

Finally, we have

Proposition 5. The independence data for $\Psi$ exceptional is,

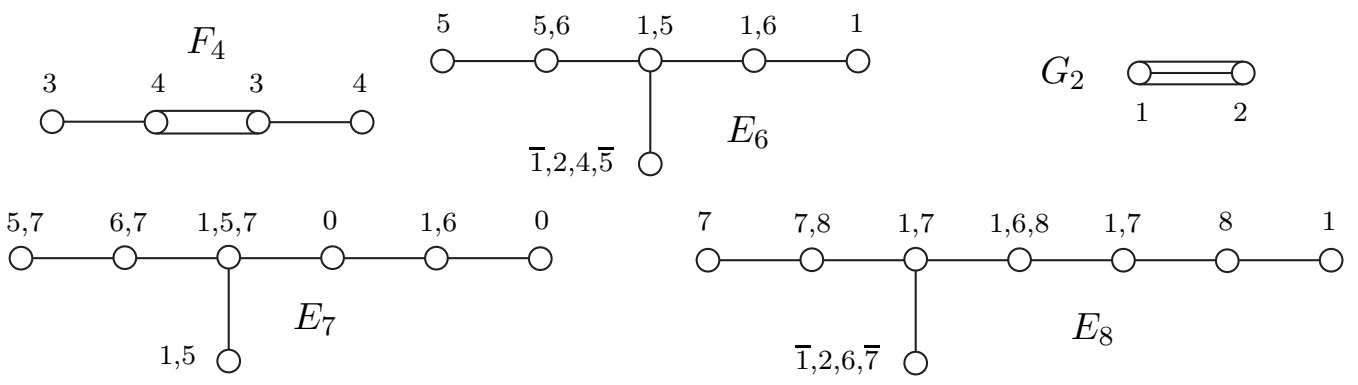

Now to another important concept. Let $W(\Psi)$ be an irreducible Weyl group and $s \in \Psi$. The pair $(\Psi, s)$ is said to be specially admissible if,

(i). in the root lattice $L$ we have $x_{s}=v_{s}$, and,

(ii). for every $t \in \Psi$ such that the minimal path from $s$ to $t$ spans a subsymbol of type $A$, we have the set $\{t\}$ is independent for $s$.

The pair is admissible if we have (i), and for every $t \in \Psi$ such that the path from $s$ to $t$ spans a subsymbol of type $A$ of $o d d$ rank, then $\{t\}$ is independent for $s$.

The first part of the definition is just an artificial device designed to exclude the pairs $\left(B_{n}, s_{n}\right)$, $\left(F_{4}, s_{3}\right),\left(F_{4}, s_{4}\right)$ and $\left(G_{2}, s_{2}\right)$, for reasons that will be explained in the proof of Theorem 5 below. It is the second part that will prove natural and useful in 93 , essentially because of the following observation: suppose that $A_{k} \subset \Psi$ is the subsymbol spanned by such a path from $s$ to $t$, with nodes $s=s_{1}, \ldots, s_{k}=t$. Then $u_{s}$ is orthogonal to $x_{2}, \ldots, x_{k}$, so is fixed by the reflections $s_{2}, \ldots, s_{k}$. As $\left\{1, s_{1}, s_{2} s_{1}, \ldots, s_{k} \cdots s_{1}\right\}$ is a set of coset representatives for $\left\langle s_{2}, \ldots, s_{k}\right\rangle$ in $W\left(A_{k}\right)$, we get that $X_{t}^{s}$ constitutes the whole $W\left(A_{k}\right)$-orbit of $\bar{u}_{s}$ in $L / 2$. Thus $T=\{t\}$ is independent for $s$ is equivalent to this $W\left(A_{k}\right)$-orbit spanning a subspace of dimension $k+1$.

Theorem 3. Let $W(\Psi)$ be a classical irreducible Weyl group. Then the admissible (respectively specially admissible) pairs $(\Psi, s)$ are given by the following, where $s=\boldsymbol{0}$ (resp. $s=0$ ),
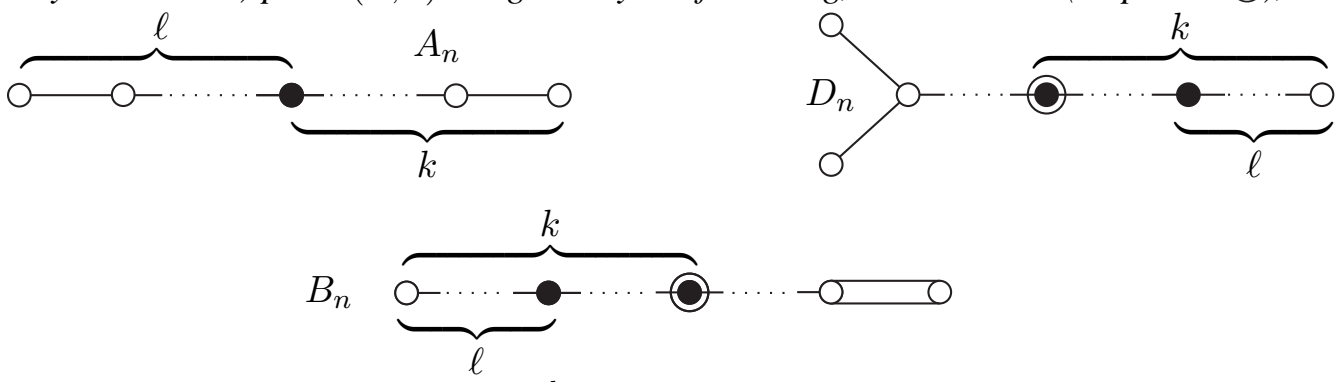

and in type $A$ we have $\ell=2^{a} m_{1}, k=2^{b} m_{2}$ with the $m_{i}$ odd, and $a<b$; in types $B$ and $D$ we have $\ell \equiv 0 \bmod 4$ and $k \equiv 2 \bmod 4$. 
The conditions in type $A$ turn out to be quite mild: if $n$ is even then all $\left(A_{n}, s\right)$ are admissible; if $n$ is odd then $k$ and $\ell$ have the same parity, and the condition becomes that they are both even, but with different 2-parts. The proof can be read straight off the diagrams in Propositions 2,5, as can the proof of,

Theorem 4. Let $W(\Psi)$ be an exceptional irreducible Weyl group. Then the admissible (resp. specially admissible) pairs $(\Psi, s)$ are given by the following, where $s=\bullet($ resp $s=0)$,

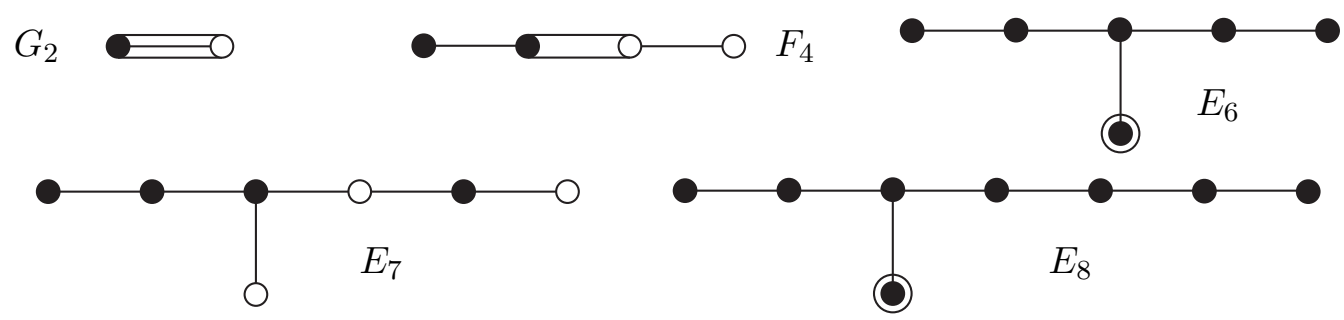

Another spin-off resulting from the independence data is the following,

Theorem 5. Let $W(\Psi)$ be an irreducible Weyl group of rank $n$ and $(\Psi, s)$ admissible. Then the subspace $\Lambda_{s} / 2 \subset L / 2$ is n-dimensional over $\mathbb{F}_{2}$.

Proof. The result follows as for any admissible pair $(\Psi, s)$ one can find a $T \subset \Psi$, with $T$ independent for $s$, and such that the union of the nodes in the minimal paths connecting $s$ to the $t \in T$ contains all but one of the nodes of $\Psi$. The pairs $\left(B_{n}, s_{n}\right),\left(F_{4}, s_{3}\right),\left(F_{4}, s_{4}\right)$ and $\left(G_{2}, s_{2}\right)$, while rather trivially satisfying condition (ii) in the definition of admissability, do not have the property of the previous sentence, hence their exclusion.

As an example, the pair $(\Psi, s)=0$ is not admissible by Theorem 3 , and for $t$ the rightmost node, $X_{s}^{t}$ is the $W(\Psi)$-orbit of $\bar{u}_{s}$, and $\Lambda_{s} / 2$ is a 1 -dimensional subspace of $L / 2$.

From now on we will only be interested in the sublattices $\Lambda_{s} \subset L$ where $(\Psi, s)$ is admissible, so that the vector spaces $\Lambda_{s} / 2 \subset L / 2$ coincide by Theorem 5 . In the rest of this section we look at some aspects of the action of the Weyl group $W(\Psi)$ on $L / 2$ as preparation for 4

To this end, let $\operatorname{End}(L / 2)$ be the endomorphism algebra of the space, writing $g(\bar{v})$ for the image of $\bar{v} \in L / 2$ under $g \in W(\Psi)$. If $g$ is an involution then $(g+1)^{2}=0 \mathrm{in} \operatorname{End}(L / 2)$, hence $\operatorname{im}(g+1) \subset \operatorname{ker}(g+1)$; moreover, $\operatorname{ker}\left(h g h^{-1}+1\right)=h \operatorname{ker}(g+1)$ and $\operatorname{im}\left(h g h^{-1}+1\right)=$ $h \mathrm{im}(g+1)$. Thus the dimension of the quotient space $\mathrm{ker}(g+1) / \mathrm{im}(g+1)$ is an invariant of the conjugacy class of the involution $g$, and we have proved the first half of,

Theorem 6. Let $W(\Psi)$ be an irreducible Weyl group with even Coxeter number $h$ and $\xi$ a Coxeter element. Then the dimension $d_{\Psi}$ of the $\mathbb{F}_{2}$ vector space

$$
\operatorname{ker}\left(\xi^{h / 2}+1\right) / \operatorname{im}\left(\xi^{h / 2}+1\right),
$$

is independent of the choice of $\xi$, with these dimensions being,

\begin{tabular}{cccccccccc}
\hline$\Psi$ & $A_{n}$ (n odd $)$ & $B_{n}$ & $D_{n}$ (n even $)$ & $D_{n}$ (n odd $)$ & $G_{2}$ & $F_{4}$ & $E_{6}$ & $E_{7}$ & $E_{8}$ \\
$d_{\Psi}$ & 1 & $n$ & $n$ & $n-2$ & 2 & 4 & 2 & 7 & 8 \\
\hline
\end{tabular}

Note that the Theorem applies to all the Weyl groups except type $A_{n}$ for $n$ even. Determining these dimensions will take a little more brute force effort; indeed we will explicitly describe the image and kernel for a particular Coxeter element, as these descriptions will prove useful in Proposition 6 below.

Proof. First and easiest, if $\Psi$ has (-1)-type, then by Proposition $1, \xi^{h / 2}=w_{\Psi}$ for any $\xi$ (as $w_{\Psi}$ is central), and $w_{\Psi}+1$ is the zero map in $\operatorname{End}(L / 2)$. Hence $\{0\}=\operatorname{im}\left(\xi^{h / 2}+1\right) \subset \operatorname{ker} \xi^{h / 2}+1=$ $L / 2$ and $d_{\Psi}=n$.

This leaves $A_{n}$ ( $n$ odd), $D_{n}$ ( $n$ odd) and $E_{6}$, for which we will consider the Coxeter element $\xi=s_{1} \ldots s_{n}$, numbering, as always, from Table 1 For type $A$, let $\left\{u_{1}, \ldots, u_{n+1}\right\}$ be the orthonormal basis realizing the isomorphism $W\left(A_{n}\right) \rightarrow \mathfrak{S}_{n+1}$ from $\$ 1.2$ Then $\xi$ corresponds to 
the $n+1$-cycle $\left(u_{1}, \ldots, u_{n+1}\right)$ and $\xi^{h / 2}$ to $\left(u_{1}, u_{\ell+1}\right)\left(u_{2}, u_{\ell+2}\right) \cdots\left(u_{\ell}, u_{n+1}\right)$ for $\ell=(n+1) / 2$. Thus with $\left\{x_{i}\right\}$ the basis for the root lattice, $\xi^{h / 2}$ interchanges $x_{i}$ and $x_{\ell+i}(i \neq \ell)$ and sends $x_{\ell}$ to $-x_{\ell}$. Thus, $\operatorname{im}\left(\xi^{h / 2}+1\right)=\left\langle\bar{x}_{i}+\bar{x}_{\ell+i} \mid 1 \leq i<\ell\right\rangle \subset L / 2$, a subspace of dimension $\ell-1$, which gives a kernel of dimension $\ell$ and hence $d_{\Psi}=1$ as claimed. It will be useful though to have an explicit description: $\operatorname{ker}\left(\xi^{h / 2}+1\right)=\operatorname{im}\left(\xi^{h / 2}+1\right) \oplus\left\langle\bar{x}_{\ell}\right\rangle$.

For type $D$ and its realisation $W\left(D_{n}\right) \rightarrow \mathfrak{S}_{\circ}^{ \pm}\left(u_{1}, \ldots, u_{n}\right)$ as the group of even signed permutations of $\left\{u_{1}, \ldots, u_{n}\right\}$, the Coxeter element $\xi$ corresponds to the even signed permutation at right. In particular, when $n$ is odd, $\operatorname{im}\left(\xi^{h / 2}+1\right)$ is the 1-dimensional space spanned by $\bar{x}_{n-1}+\bar{x}_{n}$ and $\operatorname{ker}\left(\xi^{h / 2}+1\right)=\operatorname{im}\left(\xi^{h / 2}+1\right) \oplus\left\langle\bar{x}_{1}, \ldots, \bar{x}_{n-2}\right\rangle$, giving the required $d_{\Psi}=n-2$.

Finally, $E_{6}$ really can be done by brute force, ie: by writing the generating reflections $s_{i}$ as matrices

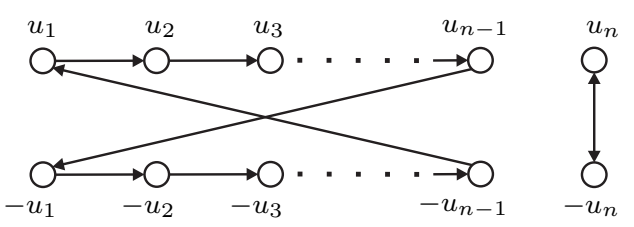
in terms of the basis $\left\{x_{i}\right\}$ for the root lattice and proceeding from there to get im $\left(\xi^{h / 2}+1\right)=$ $\left\langle\bar{x}_{1}+\bar{x}_{4}, \bar{x}_{2}+\bar{x}_{5}\right\rangle$ and $\operatorname{ker}\left(\xi^{h / 2}+1\right)=\operatorname{im}\left(\xi^{h / 2}+1\right) \oplus\left\langle\bar{x}_{1}+\bar{x}_{2}+\bar{x}_{3}, \bar{x}_{6}\right\rangle$.

We will use these descriptions in $\$$, where not the quotient, but the difference $\operatorname{ker}\left(\xi^{h / 2}+\right.$ 1) $\backslash \operatorname{im}\left(\xi^{h / 2}+1\right) \subset L / 2$ will be a target which we want to "hit" with a certain endomorphism. As is so often the case in such situations, this will be possible if the target is big enough, in fact as it transpires, if the dimension $d_{\Psi}>1$.

Proposition 6. Let $W(\Psi)$ be an irreducible Weyl group of rank $n$ with the dimension $d_{\Psi}>1$ and Coxeter number $h=2^{p} q$ where $p>0$ and $q$ odd. Then for the Coxeter element $\xi=s_{1} \ldots s_{n}$, and

$$
\alpha=1+\xi^{q}+\xi^{2 q}+\cdots+\xi^{\left(2^{p-1}-1\right) q} \in \operatorname{End}(L / 2),
$$

there is a $\bar{u} \in L / 2$ with $\alpha(\bar{u}) \in \operatorname{ker}\left(\xi^{h / 2}+1\right) \backslash i m\left(\xi^{h / 2}+1\right)$.

Proof. We proceed again on a case by case basis, starting with the $\Psi$ of $(-1)$-type, for which the difference consists of all the non-zero vectors in $L / 2$ as $d_{\Psi}=n$. Thus it suffices to show that $\alpha \neq 0$ in $\operatorname{End}(L / 2)$, and then we may choose any $\bar{u} \notin \operatorname{ker} \alpha$. Now if $p=1$, then $\alpha=1$, and we are done, leaving $\Psi=B_{n}$ and $F_{4}$ in the (-1)-type case to do.

For type $B$ and the isomorphism from $W\left(B_{n}\right)$ to the group $\mathfrak{S}^{ \pm}\left\{u_{1}, \ldots, u_{n}\right\}$ of signed permutations of the $\left\{u_{i}\right\}$ of $\oint_{1.2}$, the Coxeter element $\xi$ is the signed

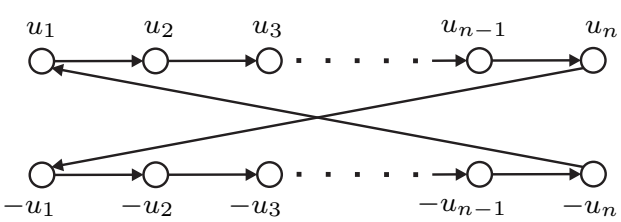
permutation at right. The root lattice $L\left(B_{n}\right)$ is spanned by the $\left\{x_{i}\right\}$ with $x_{i}=v_{i},(i<n)$ and $x_{n}=\sqrt{2} v_{n}$, and we can then read off the diagram that $\alpha\left(x_{1}\right)=x_{1}+x_{q+1}+\cdots+x_{k q+1}$ for $k=\left(2^{p-1}-1\right)$ when $q>1$, or $\alpha\left(x_{1}\right)=2\left(x_{1}+\cdots+x_{n-1}\right)+x_{n}$ when $q=1$. In either case $\alpha$ is not the zero map in $\operatorname{End}(L / 2)$. We can do $F_{4}$ by a similar brute force method to that employed for $E_{6}$ in the proof of the previous result, checking that the map $\alpha=1+\xi^{3}$ is not zero in $\operatorname{End}(L / 2)$. In type $D_{n}$ ( $n$ odd) one can check that,

$$
\alpha\left(x_{n-1}\right)=-\left(2^{p-1}-1\right)\left(x_{n-1}-x_{n}\right)-\sum_{k=1}^{2^{p-1}-1} k\left(x_{k q}+x_{k q+1}+\cdots+x_{(k+1) q-1}\right),
$$

giving $\alpha\left(\bar{x}_{n-1}\right) \in \operatorname{ker}\left(\xi^{h / 2}+1\right) \backslash \operatorname{im}\left(\xi^{h / 2}+1\right)$, while in $E_{6}$, we have $\alpha\left(\bar{x}_{1}\right)=\bar{x}_{2}+\bar{x}_{3}+\bar{x}_{4}+\bar{x}_{6}$, also hitting the target.

One can also play these games using a Coxeter element for some visible subgroup rather than all of $W(\Psi)$, and this will pay dividends later when the visible subgroup has Coxeter number with a larger 2-part. For example, $W\left(E_{6}\right)$ has $h=2^{2} 3$, whereas the visible $W\left(D_{5}\right)=$ $\left\langle s_{2}, \ldots, s_{6}\right\rangle$ has $h=2^{3}$. We then have for $\xi=s_{2} \ldots s_{6}$ that

$$
\left\langle\bar{x}_{2}+\bar{x}_{3}+\bar{x}_{5}, \bar{x}_{2}+\bar{x}_{6}\right\rangle=\operatorname{im}\left(\xi^{h / 2}+1\right) \subset \operatorname{ker}\left(\xi^{h / 2}+1\right)=\operatorname{im}\left(\xi^{h / 2}+1\right) \oplus\left\langle\bar{x}_{4}, \bar{x}_{5}\right\rangle,
$$

in $L / 2$ for $L$ the $E_{6}$ root lattice. Moreover, $\alpha=1+\xi+\xi^{2}+\xi^{3}$, gives $\alpha\left(\bar{x}_{1}\right)=\bar{x}_{3}+\bar{x}_{4}+\bar{x}_{6} \in$ $\operatorname{ker}\left(\xi^{h / 2}+1\right) \backslash \operatorname{im}\left(\xi^{h / 2}+1\right)$. 


\section{Normal torsion free subgroups}

In this section we construct, for a large class of Coxeter groups, normal torsion free subgroups whose index can be determined in terms of data associated to the Coxeter group.

Let $(W, S)$ be a Coxeter group with $S=S_{0} \cup\left\{t_{1}, \ldots, t_{m}\right\}$ and symbol $\Gamma$ at right, where $W(\Psi)=\left(W_{S_{0}}, S_{0}\right)$ is an irreducible Weyl group of rank $n$, and for each $s_{i}(1 \leq i \leq m)$, the pair $\left(\Psi, s_{i}\right)$ is admissible. Let $\sigma: W(\Psi) \rightarrow \mathrm{GL}(V)$ be the reflectional representation of the Weyl group, $L \subset V$ the root lattice, and $u_{i}=u_{s_{i}}(1 \leq i \leq m)$ the vectors in $L$ given by Lemma 2

Let $\Lambda_{i}:=\Lambda_{s_{i}}(1 \leq i \leq m)$. We will flip between the Abelian group and vector space structures on $\Lambda_{i} / 2$, with the context making clear which one we mean. Extend the $W(\Psi)$ action on $\Lambda_{i} / 2=L / 2$, to the direct product $\prod \Lambda_{i} / 2$ diagonally, ie: making $g \in W(\Psi)$ send $\left(\bar{v}_{1}, \ldots, \bar{v}_{m}\right)$ to $\left(g\left(\bar{v}_{1}\right), \ldots, g\left(\bar{v}_{m}\right)\right)$.

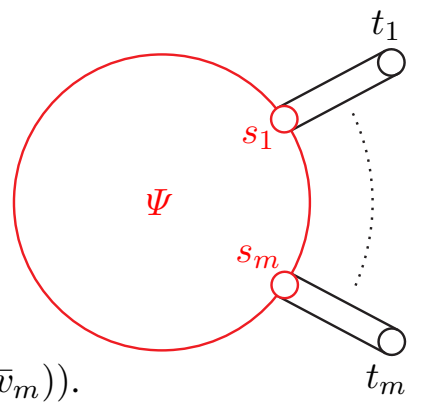
Form the semi-direct product $\prod \Lambda_{i} / 2 \rtimes W(\Psi) \cong(\mathbb{Z} / 2)^{m n} \rtimes W(\Psi)$ induced by this action, every element of which can be expressed as an ordered pair $(\mathbf{v}, g)$, where $g \in W(\Psi)$ and $\mathbf{v}=\left(\bar{v}_{1}, \ldots, \bar{v}_{m}\right)$, with $g$ and $\mathbf{v}$ (although not the $v_{i}$ ) uniquely defined. Moreover, $\prod \Lambda_{i} / 2$ is generated by the $g$-conjugates, $g \in W(\Psi)$, of the vectors, $\mathbf{u}_{i}=\left(0, \ldots, \bar{u}_{i}, \ldots, 0\right)$, with the non-zero entry in the $i$-th position, and thus $\prod \Lambda_{i} / 2 \rtimes W(\Psi)$ is generated by the $s \in S_{0}$ and the $\mathbf{u}_{i}$ (it is because of the ease of this observation that we work here with $\Lambda_{i} / 2$, rather than $L / 2$ ). Finally, the order of the semi-direct product is $2^{m n}|W(\Psi)|$, with the Weyl group order the product of the $m_{i}+1$, for $m_{i}$ the exponents in Table 2 ,

We now define a homomorphism

$$
\varphi: W(\Gamma) \rightarrow \prod \Lambda_{i} / 2 \rtimes W(\Psi),
$$

as follows: let $\varphi(s)=(\mathbf{0}, s)$ for $s \in S_{0}, \varphi\left(t_{i}\right)=\left(\mathbf{u}_{i}, 1\right)$, and extend linearly. Clearly, $\varphi\left(t_{i}\right)^{2}=$ $\left(\mathbf{u}_{i}, 1\right)^{2}=(\mathbf{0}, 1),\left(\varphi\left(t_{i}\right) \varphi\left(t_{j}\right)\right)^{2}=\left(\mathbf{u}_{i}, 1\right)\left(\mathbf{u}_{j}, 1\right)^{2}=(\mathbf{0}, 1)$ and $\left(\varphi(s) \varphi\left(s^{\prime}\right)\right)^{m_{s s^{\prime}}}=(\mathbf{0}, 1)$ for $s, s^{\prime} \in S_{0}$. Thus for $\varphi$ to be a homomorphism it remains to check that the $\left(s t_{i}\right)^{m_{s t_{i}}}=(\mathbf{0}, 1)$ relations are satisfied in the image for $s \in S_{0}$. As the $u_{i}$ are orthogonal to the $v_{s}$ for $s \in S_{0} \backslash\left\{s_{i}\right\}$, we have $\mathbf{u}_{i}^{s}:=s \mathbf{u}_{i} s^{-1}=\mathbf{u}_{i}$ in the semi-direct product, hence

$$
\left((\mathbf{0}, s)\left(\mathbf{u}_{i}, 1\right)\right)^{2}=\left(\mathbf{u}_{i}+\mathbf{u}_{i}^{s}, 1\right)=(\mathbf{0}, 1),
$$

for these $s$. Finally, for $1 \leq i \leq m$,

$$
\left(\left(\mathbf{0}, s_{i}\right)\left(\mathbf{u}_{i}, 1\right)\right)^{4}=\left(2 \mathbf{u}_{i}+2 \mathbf{u}_{i}^{s}, 1\right)=(\mathbf{0}, 1),
$$

and so $\varphi$ is a homomorphism, surjective as the semi-direct product is generated by the images of the $s \in S_{0}$ and the $t_{i}$.

We now show that when the pairs $\left(\Psi, s_{i}\right)$ are specially admissible, the kernel of $\varphi$ is torsion free, otherwise, if some are merely admissible, then it is almost torsion free, and the offending torsion can be pinpointed pretty accurately. The first step is to observe that if $\Delta_{1}, \Delta_{2} \subset \Gamma$ are disjoint subsymbols, then the $\varphi W\left(\Delta_{i}\right)$ intersect trivially in $\prod \Lambda_{i} / 2 \rtimes W(\Psi)$. For, if $\Delta \subset \Gamma$ is a subsymbol and $g \in W(\Delta)$, then $\varphi(g)=\left(\mathbf{v}, g^{\prime}\right)$ where $g^{\prime} \in W(\Delta \cap \Psi)$, and $\mathbf{v} \in \prod \Lambda_{i} / 2$ has $i$-th coordinate non-zero only if $t_{i} \in \Delta$. The result now follows as the visible subgroups $W\left(\Delta_{i}\right)$ intersect trivially, and by the uniqueness of the $(\mathbf{v}, g)$ expression for elements of $\prod \Lambda_{i} / 2 \rtimes$ $W(\Psi)$.

In particular, by the comments following Theorem 1 if $\varphi$ is faithful when restricted to disjoint visible subgroups $W\left(\Delta_{1}\right), W\left(\Delta_{2}\right)$, then it is also faithful on $W\left(\Delta_{1} \amalg \Delta_{2}\right)$, and thus to show that $\operatorname{ker} \varphi$ is torsion free, it suffices to show that $\varphi$ is faithful on the finite visible $W(\Delta)$, for connected $\Delta \subset \Gamma$.

Before proceeding, we mention in passing that one may be tempted by a simpler choice for $\varphi$, namely letting $\Lambda$ be the lattice spanned by the $W(\Psi)$-orbit of all the $\left\{u_{1}, \ldots, u_{m}\right\}$ simultaneously, forming $\Lambda / 2 \rtimes W(\Psi)$ and defining $\varphi(s)=(0, s)$ and $\varphi\left(t_{i}\right)=\left(u_{i}, 1\right)$. It turns out that 
this $\varphi$ is also faithful on the finite visible $W(\Delta)$ for $\Delta \subset \Gamma$ connected, but not generally on the finite visibles, because $\varphi W\left(\Delta_{i}\right)$ with disjoint $\Delta_{i}$ can have non-trivial intersection. For example, if $\Psi$ is of type $E_{6}$ with the two admissible nodes,

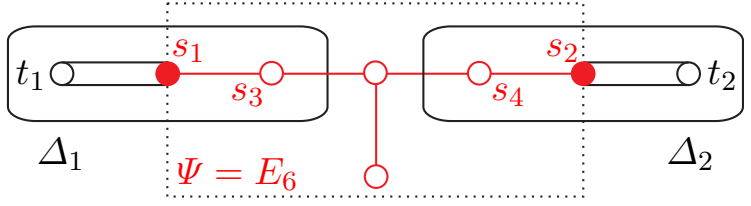

then for $i=1,2$, both $1 \neq\left(s_{i} t_{i} s_{i+2}\right)^{3} \in W\left(\Delta_{i}\right)$ map via this $\varphi$ to the same non-trivial element of $\Lambda / 2 \rtimes W(\Psi)$.

Lemma 3. Let $B_{k} \subset \Gamma$ contain one (hence exactly one) of the $t_{i}$,

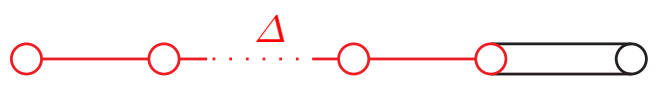

Then $\varphi$ is faithful on $W\left(B_{k}\right)$ if and only if the $W(\Delta)$-orbit of $\bar{u}_{i} \in L / 2$ spans a subspace $U_{i} \subset L / 2$ of dimension $k$.

In particular, by the comments following the definition of admissibility in $\$ 2$ if the pair $\left(\Psi, s_{i}\right)$ is admissible (respectively specially admissible) then $\varphi$ is faithful on the visible $W\left(B_{k}\right)$ with $t_{i} \in B_{k}$ and $k$ even (resp. for all $k$ ). The result follows since $W\left(B_{k}\right) \cong(\mathbb{Z} / 2)^{k} \rtimes W(\Delta)$, whereas, $\varphi W\left(B_{k}\right) \cong U_{i} \rtimes W(\Delta)$, thinking of $U_{i}$ as an Abelian group.

We are now ready for,

Theorem 7. Let $\Gamma$ be the symbol $(\dagger)$ with $W(\Psi)$ an irreducible Weyl group of rank $n$ and exponents $m_{i}$, and the pairs $\left(\Psi, s_{i}\right)$ specially admissible for all $i$. Then ker $\varphi \subset W(\Gamma)$ is a torsion free subgroup of index

$$
[W(\Gamma): \operatorname{ker} \varphi]=2^{m n} \prod_{i=1}^{n}\left(m_{i}+1\right) .
$$

Proof. We have observed that it suffices to show that $\varphi$ is faithful on the finite visible $W(\Delta)$ for connected $\Delta \subset \Gamma$. This follows immediately for $\Delta \subset \Psi$, and if $\Delta=\left\{t_{i}\right\}$, then the faithfulness follows as $\bar{u}_{i} \neq 0$. The remaining possibility is that $\Delta$ is of type $B_{k}$ for $k>1$ and containing (exactly) one of the $t_{i}$ generators and this case follows by special admissibility and the comments following Lemma 3 .

If some of the pairs are admissible but not specially admissible, we get elements of finite order in $\operatorname{ker} \varphi$, but we can say where, and what they look like:

Proposition 7. Let $\Gamma$ be the symbol $(\dagger)$ with the pairs $\left(\Psi, s_{i}\right)$ admissible, and let $W(\Delta)$ be finite visible with $\Delta \subset \Gamma$ connected. Then ker $\varphi \subset W(\Gamma)$ contains a non-trivial element $g \in W(\Delta)$ of prime order if and only if there is $\Upsilon=\coprod \Delta_{i} \subset \Delta$ with the $\Delta_{i}$ connected, and

(i). $\Delta_{1}$ of type $B_{k}$ for $k$ odd, and with a $t_{i} \in \Delta_{1}$ for some (hence exactly one) $i$;

(ii). $\Delta_{i}$ is of (-1)-type and contained in $\Psi$ for $i>1$;

(iii). $g$ is conjugate to the element of longest length in $W(\Upsilon)$.

Proof. As in the proof of Theorem 7 we can reduce to the case that $\Delta$ has type $B$ and contains (exactly) one of the $t_{i}$ generators. Any element of prime order $p>2$ in $W(\Delta)$ is conjugate by Lemma 1 to an element in $W(\Delta \cap \Psi)$. As $\operatorname{ker} \varphi$ contains no such elements, it can only contain prime order elements of order 2 . By Theorem 2 any such $g$ is conjugate to the element of longest length in a finite visible subgroup of (-1)-type, ie: there is $\Upsilon=\coprod \Delta_{i} \subset \Delta$ with the $\Delta_{i}$ connected and of (-1)-type, and with $w_{\Upsilon} \in \operatorname{ker} \varphi$ for $w_{\Upsilon}$ the element of longest length in $W(\Upsilon)$. 
If all the $\Delta_{i} \subset \Psi$ then $\varphi$ is faithful on the $W\left(\Delta_{i}\right)$, hence on the $W(\Upsilon)$, so we must have one of the $t_{i}$ contained in $\Delta_{1}$, say, and thus $\Delta_{1}$ of type $B_{k}$. As $\Delta$ is connected we must also have the $\Delta_{i} \subset \Psi$ for $i>1$. If $k$ is even, then $\varphi$ is faithful on $W\left(\Delta_{1}\right)$ by the admissibility of $\left(\Psi, s_{1}\right)$ and Lemma 3, and hence on $W(\Upsilon)$. Thus, $\Delta_{1}=B_{k}$ for $k$ odd as required.

In order to get normal torsion free subgroups when some of the pairs are admissible but not specially admissible, we need to re-jig $\varphi$ a little to handle the torsion described in the Proposition. The idea is simple enough: we delve deeper into $\operatorname{ker} \varphi$ to avoid the elements of longest length in type $B$ groups of odd rank. To this end, fix $i$ and let $g \in W(\Gamma)$ and $w$ a word representing it that contains $\lambda_{g}^{i}$ occurrences of the generator $t_{i}$. Define a map $f_{i}: S \rightarrow \mathbb{Z} / 2$ that sends $t_{i}$ to 0 and $s \neq t_{i}$ to 1 . As the Coxeter relations are satisfied in $\mathbb{Z}_{2}$ by the $f_{i}(S)$, the map extends to a homomorphism $W \rightarrow \mathbb{Z} / 2$, with $f_{i}(g)=\lambda_{g}^{i}(\bmod 2)$. Thus the parity $\lambda_{g}^{i}(\bmod 2)$ of $\lambda_{g}^{i}$ is independent of the word $w$. Observe that as

$$
w_{B_{n}}=\left(s_{1} s_{2} \cdots s_{n-1} s_{n} s_{n-1} \cdots s_{2} s_{1}\right) \cdots\left(s_{n-2} s_{n-1} s_{n} s_{n-1} s_{n-2}\right) \cdot\left(s_{n-1} s_{n} s_{n-1}\right) \cdot s_{n},
$$

we have $f_{i}\left(w_{B_{n}}\right)=n(\bmod 2)$.

Let the nodes $\left\{s_{i}\right\}$ giving the admissible pairs be divided $\left\{s_{1}, \ldots, s_{\ell}, s_{\ell+1}, \ldots, s_{m}\right\}$, with the first $\ell$ admissible but not specially admissible and the remaining specially admissible. Define

$$
\varepsilon: W(\Gamma) \rightarrow \mathbb{Z} / 2^{\ell}:=\prod_{\ell \text { copies }} \mathbb{Z} / 2
$$

by $\varepsilon(g)=\mathbf{x}_{g}=\left(f_{1}(g), \ldots, f_{\ell}(g)\right) \in \mathbb{Z} / 2^{\ell}$, the $f_{i}$ as above, a product of homomorphisms, hence a homomorphism, and moreover, constant on each conjugacy class of $W(\Gamma)$. Now define

$$
\widehat{\varphi}: W(\Gamma) \rightarrow \mathbb{Z} / 2^{\ell} \times\left(\prod \Lambda_{i} / 2 \rtimes W(\Psi)\right),
$$

by $\widehat{\varphi}=\varepsilon \times \varphi$, ie: $\widehat{\varphi}(g)=(\varepsilon(g), \varphi(g))$, also a homomorphism.

For each $\left(\Psi, s_{i}\right), 1 \leq i \leq \ell$, there must be a subsymbol $B_{k} \subset \Gamma$ of odd rank $k$ containing the Coxeter generator $t_{i}$, and with $\varphi$ not faithful on $W\left(B_{k}\right)$, since the pair is admissible but not specially admissible. In particular, $\operatorname{ker} \varphi$ contains the element $w_{i}=w_{\Upsilon}$ of longest length in $W(\Upsilon)$ with $\Upsilon=\coprod \Delta_{j} \subset B_{k}$ and $\Delta_{1}$ also a type $B$ of odd rank by Proposition 7, Then by (4), $f_{i}\left(w_{i}\right)=1$, and $f_{j}\left(w_{i}\right)=0$ for $j \neq i$. The result is that $\varphi\left(w_{i}\right)=(\mathbf{0}, 1)$ and

$$
\varepsilon\left(w_{i}\right)=\mathbf{x}_{i}=(0, \ldots, 1, \ldots, 0),
$$

with the 1 in the $i$-th position. Thus, $\widehat{\varphi}\left(w_{i}\right)=\left(\mathbf{x}_{i}, \mathbf{0}, 1\right)$, and we already have that $\widehat{\varphi}\left(s \in S_{0}\right)=$ $(\mathbf{0}, \mathbf{0}, s)$ and $\widehat{\varphi}\left(t_{i}\right)=\left(\mathbf{x}_{i}, \mathbf{u}_{i}, 1\right)$, so that $\widehat{\varphi}\left(w_{i} t_{i}\right)=\left(\mathbf{0}, \mathbf{u}_{i}, 1\right)$. As the group $\mathbb{Z} / 2^{\ell} \times\left(\prod \Lambda_{i} / 2 \rtimes\right.$ $W(\Psi))$ is generated by the $\left(\mathbf{x}_{i}, \mathbf{0}, 1\right),(\mathbf{0}, \mathbf{0}, s)$ and $\left(\mathbf{0}, \mathbf{u}_{i}, 1\right)$, we get that $\widehat{\varphi}$ is surjective.

If $\Delta \subset \Gamma$, then the image under $\widehat{\varphi}$ of $g \in W(\Delta)$ is $\left(\mathbf{x}_{g}, \mathbf{v}, g^{\prime}\right)$ with $g^{\prime} \in W(\Delta \cap \Psi)$, and the $\mathbf{v} \in \prod \Lambda_{i} / 2$ and $\mathbf{x}_{g} \in \mathbb{Z} / 2^{\ell}$ having $i$-th coordinate non-zero only if $t_{i} \in \Delta$. Thus, $\widehat{\varphi}$, like $\varphi$, sends trivially intersecting visibles to trivially intersecting subgroups, so that if $\widehat{\varphi}$ is faithful on $W\left(\Delta_{i}\right)$ with the $\Delta_{i} \subset \Gamma$ disjoint, then it is also faithful on $W\left(\Delta_{1} \coprod \Delta_{2}\right)$.

Theorem 8. Let $\Gamma$ be the symbol $(\dagger)$ with $W(\Psi)$ an irreducible Weyl group of rank $n$ and exponents $m_{i}$, and with the pairs $\left(\Psi, s_{i}\right),(1 \leq i \leq \ell)$ admissible but not specially admissible and the remaining pairs specially admissible. Then ker $\widehat{\varphi} \subset W(\Gamma)$ is a torsion free subgroup of index

$$
[W(\Gamma): \operatorname{ker} \widehat{\varphi}]=2^{m n+\ell} \prod_{i=1}^{n}\left(m_{i}+1\right) .
$$

Proof. It suffices as before to show that $\widehat{\varphi}$ is faithful on the finite visible $W(\Delta)$ with $\Delta$ connected, which in turn follows if ker $\widehat{\varphi}$ contains no elements of prime order from such subgroups. Such an element $g \in \operatorname{ker} \widehat{\varphi}$ is contained in both $\operatorname{ker} \varepsilon$ and $\operatorname{ker} \varphi$, and the second of these, combined with Proposition 7 gives that $g$ is conjugate to the element $w_{\Upsilon}$ of longest length in $W(\Upsilon)$ where the $\Upsilon=\coprod \Delta_{j}$ have the properties described in the Proposition. As $\varepsilon$ is constant on the conjugacy classes we have $w_{\Upsilon} \in \operatorname{ker} \varepsilon$ also. But $\lambda_{w_{\Upsilon}}^{i}=1$, as the element of longest length in a type $B$ group of odd rank contains an odd number of occurrences of $t_{i}$ and thus $\varepsilon\left(w_{\Upsilon}\right) \neq \mathbf{0}$. This contradiction gives the result. 


\section{Even bigger, non-normal, torsion free subgroups}

In the last section we constructed normal torsion free subgroups in our family of Coxeter groups; in this, we extend them to larger non-normal torsion free subgroups. The strategy is simple: we look for subgroups $H$ in the finite image $W(\Gamma) / \operatorname{ker} \theta$, where $\theta=\varphi$ or $\widehat{\varphi}$, and with the property that no non-trivial element of finite order in $W(\Gamma)$ maps via $\theta$ into $H$. Thus, $\theta^{-1} H \subset W(\Gamma)$ will be a torsion free subgroup containing $\operatorname{ker} \theta$, but with $1 /|H|$-th the index. Moreover, both the normality of the original group and the construction of the new one will have geometrical interpretations in the next section.

For simplicity we restrict our attention to cyclic $H$ of order $2^{p}$ for some $p$, and work just with $\widehat{\varphi}$, pointing out where appropriate the corresponding results for $\varphi$. Thus, with $\widehat{\varphi}: W(\Gamma) \rightarrow$ $\mathbb{Z} / 2^{\ell} \times\left(\prod \Lambda_{i} / 2 \rtimes W(\Psi)\right)$ from $\S$, we seek an

$$
H=\mathbb{Z} /\left(2^{p}\right) \subset \mathbb{Z} / 2^{\ell} \times\left(\prod \Lambda_{i} / 2 \rtimes W(\Psi)\right),
$$

with $\widehat{\varphi}^{-1} H \subset W(\Gamma)$ torsion free.

Firstly, if $\widehat{\varphi}^{-1} H$ contains non-trivial torsion, then it contains 2 -torsion: if $g \in \widehat{\varphi}^{-1} \mathbb{Z} /\left(2^{p}\right)$ has finite order then as $\widehat{\varphi}$ kills no torsion in $W(\Gamma)$, the image $\widehat{\varphi}(g)$ has the same order in $\mathbb{Z} /\left(2^{p}\right)$, and thus $g$ has even order; some power of $g$ thus has order 2 , with order 2 image in $\mathbb{Z} /\left(2^{p}\right)$. We conclude that $\widehat{\varphi}^{-1} \mathbb{Z} /\left(2^{p}\right)$ is torsion free if and only if there are no elements of order 2 in $W(\Gamma)$ mapping via $\widehat{\varphi}$ to $H$; alternatively, $H$ intersects trivially any conjugacy class of order two in $\mathbb{Z} / 2^{\ell} \times\left(\prod \Lambda_{i} / 2 \rtimes W(\Psi)\right)$ that is the image of a conjugacy class of elements of order 2 in $W(\Gamma)$.

Thus, our efforts will be focused on the conjugacy classes of elements of order 2 in $W(\Gamma)$ and $W(\Gamma) / \operatorname{ker} \widehat{\varphi}$, as well as the relationship between them given by $\widehat{\varphi}$. Let $\mathcal{J}_{\Gamma}$ be the $W(\Gamma)$ equivalence classes of subsymbols of (-1)-type from $\$ 1.3$ and for $\Delta \in \mathcal{J}_{\Gamma}$, let $w_{\Delta}$ be the element of longest length in $W(\Delta)$. Although the empty symbol is not of $(-1)$-type, we adopt the convention $w_{\varnothing}=1$. If $\Delta \in \mathcal{J}_{\Gamma}$, write $\mathrm{C}(\Delta)$ for the conjugacy class of $w_{\Delta}$ in $W(\Delta)$.

We can get a very rough parametrization of the conjugacy classes 2 -torsion in the image group. First, we extend the notation of $\oint_{2}$, so that if $\alpha \in \operatorname{End}\left(\prod \Lambda_{i} / 2=\prod L / 2\right)$, then $\operatorname{ker} \alpha$ and $\operatorname{im} \alpha$ are the direct sums of the kernels and images in $\operatorname{End}\left(\Lambda_{i} / 2\right)$. Let $\mathcal{T}$ be the set of triples $(\mathbf{x}, \mathbf{v}, \Delta)$ where $\mathbf{x} \in \mathbb{Z} / 2^{\ell}, \mathbf{v} \in \prod \Lambda_{i} / 2$ and $\Delta \in \mathcal{J}_{\Psi} \cup\{\varnothing\}$.

Lemma 4. The map $(\mathbf{x}, \mathbf{v}, \Delta) \mapsto\left(\mathbf{x}, \mathbf{v}, w_{\Delta}\right)$ induces a surjection from $\mathcal{T}$ to the set of conjugacy classes of involutions in the group $\mathbb{Z} / 2^{\ell} \times\left(\prod \Lambda_{i} / 2 \rtimes W(\Psi)\right)$. Writing $\mathrm{C}(\mathbf{x}, \mathbf{v}, \Delta)$ for the class that is the image of $(\mathbf{x}, \mathbf{v}, \Delta) \in \mathcal{T}$, we have

(i). if $\mathcal{C}\left(\mathbf{x}_{1}, \mathbf{v}_{1}, \Delta_{1}\right)=\mathfrak{C}\left(\mathbf{x}_{2}, \mathbf{v}_{2}, \Delta_{2}\right)$ then $\mathbf{x}_{1}=\mathbf{x}_{2}$ and $\Delta_{1}=\Delta_{2}$;

(ii). the class $\mathcal{C}(\mathbf{0}, \mathbf{0}, \Delta)=\bigcup\{(\mathbf{0}, \mathbf{u}, g) \mid \mathbf{u} \in$ im $(g+1)\}$, where the union is over all $W(\Psi)$ conjugates $g$ of $w_{\Delta}$.

Proof. If $(\mathbf{x}, \mathbf{v}, g) \in \mathbb{Z} / 2^{\ell} \times\left(\prod \Lambda_{i} / 2 \rtimes W(\Psi)\right)$, then $(\mathbf{x}, \mathbf{v}, g)^{2}=\left(\mathbf{0},(g+1) \mathbf{v}, g^{2}\right)$, so that the element is an involution if and only if either $g$ is the identity or $g$ is an involution and $\mathbf{v} \in \operatorname{ker}(g+1)$. Thus every involution $(\mathbf{x}, \mathbf{u}, g)$ is conjugate to a $\left(\mathbf{x}, \mathbf{v}, w_{\Delta}\right)$ with the corresponding triple $(\mathbf{x}, \mathbf{v}, \Delta) \in \mathcal{T}$, thus giving the surjectivity of the map. Clearly elements $\left(\mathbf{x}_{1}, \mathbf{v}_{1}, g_{1}\right)$ and $\left(\mathbf{x}_{1}, \mathbf{v}_{2}, g_{2}\right)$ are conjugate only if $\mathbf{x}_{1}=\mathbf{x}_{2}$ and the $g_{i}$ are conjugate in $W(\Psi)$, and this gives the first part. The second part follows as $(\mathbf{x}, \mathbf{u}, h)\left(\mathbf{0}, \mathbf{0}, w_{\Delta}\right)(\mathbf{x}, \mathbf{u}, h)^{-1}=(\mathbf{0},(1+$ $\left.\left.h w_{\Delta} h^{-1}\right)(\mathbf{u}), h w_{\Delta} h^{-1}\right)$.

When the image group is $W(\Gamma) / \operatorname{ker} \varphi$, the above goes straight through with pairs $(\mathbf{v}, \Delta)$ instead.

Lemma 5. Let $\Theta \in \mathcal{J}_{\Gamma}$.

(i). $\Theta$ is the disjoint union of a $\Delta^{\prime} \subset \Psi$ with symbols of type $B$ containing $a t_{i}$, and $\widehat{\varphi} \mathcal{C}(\Theta)=$ $\mathrm{e}\left(\mathbf{x}, \mathbf{v}, \Delta^{\prime}\right)$ for some $\mathbf{x}, \mathbf{v}$. 
(ii). Let $\Delta \in \mathcal{J}_{\Psi}$ be of maximal rank. If $\widehat{\varphi} \mathrm{C}(\Theta)=\mathcal{C}(\mathbf{x}, \mathbf{v}, \Delta)$ for some $\mathbf{x}, \mathbf{v}$, then $\Theta$ is a disjoint union of a $\Delta^{\prime}$ that is $W(\Psi)$-equivalent to $\Delta$ together with some of the nodes $t_{i}$ where $s_{i} \notin \Delta^{\prime}$. (iii). If $\mathrm{C}(\mathbf{0}, \mathbf{v}, \Delta)=\widehat{\varphi} \mathrm{C}(\Theta)$ for $\Delta \in \mathcal{J}_{\Psi}$ of maximal rank, then $\mathrm{C}(\mathbf{0}, \mathbf{v}, \Delta)=\mathcal{C}(\mathbf{0}, \mathbf{0}, \Delta)$.

It is the last part that is the really useful bit: only one of the classes of the form $\mathcal{C}(\mathbf{0}, \mathbf{v}, \Delta)$, namely $\mathrm{C}(\mathbf{0}, \mathbf{0}, \Delta)$, lies in the image of the 2 -torsion of $W(\Gamma)$, when $\Delta$ is of maximal rank.

Proof. If $\Theta \in \mathcal{J}_{\Gamma}$ is connected and $\not \subset \Psi$ then it contains one (hence exactly one) of the $t_{i}$ and so is of type $B$, giving the first part of (i). Observe that the image under $\widehat{\varphi}$ of the $w_{B_{n}}$ given by (4) collapses to the identity in the third component, and since $w_{\Theta}$ is the product of the elements of longest length in the irreducible components of $W(\Theta)$, we get $\widehat{\varphi}\left(w_{\Theta}\right)=\left(\mathbf{x}, \mathbf{v}, w_{\Delta^{\prime}}\right)$ for some $\mathbf{x}$ and $\mathbf{v}$ as claimed.

In part (ii), $\Theta$ must now be the disjoint union of a $\Delta^{\prime}$ that is $W(\Psi)$-equivalent to $\Delta$ and some type $B$ 's that contain various $t_{i}$ 's. By checking through the possible $\Psi$ in Table 1 and the $\Delta \subset \Psi$ with $\Delta \in \mathcal{J}_{\Psi}$ of maximal rank, we can see that every $s \in \Psi$ not already in $\Delta$ is joined by an edge to it. Thus, the only type $B$ 's that remain disjoint from $\Delta$ are the single nodes $t_{i}$ with $s_{i} \notin \Delta^{\prime}$.

Now, for $\Theta$ of the form given in part (ii), the element $w_{\Theta}$ is the product of $w_{\Delta^{\prime}}$ with some of the $t_{i}$ 's such that $s_{i} \notin \Delta^{\prime}$. These $s_{i}$ must be admissible but not specially admissible, as for every specially admissible pair $(\Psi, s)$ we have $s \in \Delta^{\prime}$ by Theorems 3 . 5 Thus $\widehat{\varphi}\left(w_{\Theta}\right)=\left(\mathbf{x}, \mathbf{v}, w_{\Delta}\right)$, where $\mathrm{x}$ contains a 1 in the $i$-th coordinate for each $t_{i}$ present, by the comments preceeding Theorem 8 . Since by Lemma 4] $\mathcal{C}(\mathbf{x}, \mathbf{v}, \Delta) \neq \mathcal{C}(\mathbf{0}, \mathbf{v}, \Delta)$ when $\mathbf{x} \neq \mathbf{0}$, the only $\mathcal{C}(\Theta)$ mapping to a $\mathrm{C}(\mathbf{0}, \mathbf{v}, \Delta)$ is $\mathrm{C}\left(\Delta^{\prime}\right)=\mathrm{C}(\Delta)$ itself, for which we have $\widehat{\varphi} \mathfrak{C}(\Delta)=\mathcal{C}(\mathbf{0}, \mathbf{0}, \Delta)$.

If we have $\varphi$ instead, then the pairs $\left(\Psi, s_{i}\right)$ must all be specially admissible, so that if $\varphi \mathrm{C}(\Theta)=\mathrm{C}(\mathbf{v}, \Delta)$ for $\Delta$ maximal, then again $\mathrm{C}(\Theta)=\mathcal{C}(\Delta)$, for which $\varphi \mathrm{C}(\Delta)=\mathfrak{C}(\mathbf{0}, \Delta)$. Here is the main result of the section:

Theorem 9. Let $W(\Gamma)$ be the Coxeter group $(\dagger)$, where $W(\Psi)$ is an irreducible Weyl group of rank $n$, with exponents $m_{i}$, the dimension $d_{\Psi}>1$, Coxeter number $h=2^{p} q$ where $p>0$ and $q$ odd, the first $\ell$ pairs $\left(\Psi, s_{i}\right)$ admissible and the remaining ones specially admissible. Then there is a Coxeter element $\xi \in W(\Psi)$, an $\mathbf{x} \in \mathbb{Z} / 2^{\ell}$ and $a \mathbf{u} \in \prod \Lambda_{i} / 2$, such that $\zeta=\left(\mathbf{x}, \mathbf{u}, \xi^{q}\right)$ generates a subgroup,

$$
\langle\zeta\rangle \cong \mathbb{Z} /\left(2^{p}\right) \subset W(\Gamma) / \operatorname{ker} \widehat{\varphi},
$$

with $\widehat{\varphi}^{-1}\langle\zeta\rangle \subset W(\Gamma)$ torsion free of index $2^{m n+\ell-p} \prod_{i=1}^{n}\left(m_{i}+1\right)$.

The evenness of the Coxeter number rules out the type $A$ of even rank and the dimension $d_{\Psi}>1$ rules out the type $A$ 's of odd rank. Everything else is ruled in.

Proof. We have $\zeta^{k}=\left(k \mathbf{x}, \mathbf{u}+\xi^{q}(\mathbf{u})+\xi^{2 q}(\mathbf{u})+\cdots+\xi^{(k-1) q}(\mathbf{u}), \xi^{k q}\right)$. Let $\xi=s_{1} \ldots s_{n}, \mathbf{x}=\mathbf{0}$ and $\mathbf{u}=(\bar{u}, \ldots, \bar{u})$ where $\bar{u} \in L / 2$ is given by Proposition 6 Then $\zeta^{2^{p-1}}$ has middle component $\alpha(\mathbf{u})$ for the $\alpha$ of Proposition 6 and last component $\xi^{\left(2^{p-1}\right) q}=\xi^{h / 2}$. Since $\alpha(\mathbf{u}) \in \operatorname{ker}\left(\xi^{h / 2}+1\right)$

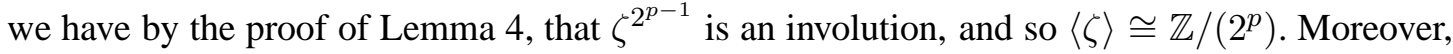
by Proposition 1, this involution lies in $\mathcal{C}(\mathbf{0}, \alpha(\mathbf{u}), \Delta)$, where $\Delta \in \mathcal{J}_{\Psi}$ is of maximal rank, and by Lemma 5 (iii), $\widehat{\varphi}^{-1}\langle\zeta\rangle$ is torsion free if $\mathcal{C}(\mathbf{0}, \alpha(\mathbf{u}), \Delta) \neq \mathcal{C}(\mathbf{0}, \mathbf{0}, \Delta)$. But one last application of Proposition 6, together with Lemma 4(ii), gives $\zeta^{2^{p-1}} \notin \mathcal{C}(\mathbf{0}, \mathbf{0}, \Delta)$, and we are done.

In the case where all the $\left(\Psi, s_{i}\right)$ are specially admissible, the Theorem remains unchanged if $\widehat{\varphi}$ is replaced by $\varphi$ (and the references to $\mathrm{x}$ and $\mathbb{Z} / 2^{\ell}$ removed of course) Finally, we wrap-up with an omnibus result,

Theorem 10. Let $W(\Gamma)$ be the Coxeter group with symbol $(\dagger)$, where

(i). $\Psi=A_{n}$ with $n$ odd. Then the element $\zeta=\left(\mathbf{0}, \mathbf{u}, \xi^{h / 2}\right)$ for some $\mathbf{u}$ and $\xi=s_{1} \ldots s_{n}$, generates a subgroup $\cong \mathbb{Z} / 2$ with $\widehat{\varphi}^{-1}\langle\zeta\rangle$ torsion free.

(ii). $\Psi=E_{6}$. Then if $\xi=s_{2} \ldots s_{n}$ is a Coxeter element of the visible $W\left(D_{5}\right)=\left\langle s_{2}, \ldots, s_{6}\right\rangle$, the element $\zeta=(\mathbf{0}, \mathbf{u}, \xi)$ for some $\mathbf{u}$ generates a subgroup $\cong \mathbb{Z} /\left(2^{3}\right)$ with $\widehat{\varphi}^{-1}\langle\zeta\rangle$ torsion free. 
Notice that while $E_{6}$ does in fact fall under the auspices of Theorem 9 , the torsion free subgroup given there has twice the index as that given above. The proof of Theorem 10 is entirely analogous to Theorem 9 , and uses the relevant calculations from the end of $₫ 2$.

\section{Geometric manifolds and their symmetries}

Let $X$ be one of the three simply connected Riemannian $n$-manifolds of constant sectional curvature, ie: $X=$ the $n$-sphere $S^{n}$, the Euclidean space $\mathbb{E}^{n}$, or the $n$-dimensional hyperbolic space $\mathbb{H}^{n}$. Let Isom $X$ be the group of isometries of $X$ and $G \subset$ Isom $X$ a discrete subgroup. Then $G$ acts properly discontinuously on $X$, and in the presence of curvature (ie: when $X \neq \mathbb{E}^{n}$ ), the volume of a fundamental region is an invariant for $G$, the covolume covol $(G)$.

If $G_{1} \subset G_{2} \subset$ Isom $X$ are discrete, then $M=X / G_{1}$ is a complete Riemannian $n$-manifold with constant sectional curvature that of $X$ if and only if $G_{1}$ acts freely. In this case $G_{1} \cong \pi_{1}(M)$ and $M$ has volume vol $(M)=\left[G_{2}: G_{1}\right] \operatorname{covol}\left(G_{2}\right)$. Moreover, we have a free action whenever $G$ is torsion free (the converse is not true when Isom $X$ is compact, ie: when $X=S^{n}$, where discrete subgroups are necessarily finite, but there still exist free actions of non-trivial groups).

An automorphism of $M=X / G$ is an isometry $M \rightarrow M$, and the group $\operatorname{Aut}(M)$ of all such is isomorphic to $N(G) / G$, where $N(G)$ is the normalizer in Isom $X$ of $G$. A subgroup $H \subset$ $\operatorname{Aut}(M)$ acts freely on $M$ precisely when $G \triangleleft \eta^{-1} H$ is torsion free, for $\eta: N(G) \rightarrow N(G) / G$ the quotient homomorphism. Letting $\widehat{M}=X / \eta^{-1} H$, then gives a Galois covering of manifolds $M \rightarrow \widehat{M}$ with group of deck transformations $H$.

Suppose now that $G$ is (isomorphic to) a Coxeter group $W(\Gamma)$. Then $W(\Gamma)$ is finite when $X=S^{n}$, an affine Weyl group (as in eg: [17, Chapter 4]) when $X=\mathbb{E}^{n}$, and hyperbolic in the sense of $₫ 1.1$ when $X=\mathbb{H}^{n}$.

Looking more closely at $X=\mathbb{H}^{n}$, there is no general method for computing the covolumes of hyperbolic Coxeter groups. If the fundamental region is a polytope with a particularly simple structure, such as a simplex, then the covolumes have been computed [18]. If $n$ is even, one can instead appeal to homological considerations, for the Gauss-Bonnet(-Hirzebruch) formula [15, 28], gives

$$
\operatorname{covol} W(\Gamma)=\kappa_{n} \chi W(\Gamma), \text { where } \kappa_{n}=2^{n}(n !)^{-1}(-\pi)^{n / 2}(n / 2) !
$$

and $\chi W(\Gamma)=\sum_{i}(-1)^{i}$ rank $_{\mathbb{Z}} H_{i} W(\Gamma)$ is the (group) Euler characteristic of $W(\Gamma)$. By [5], the computation of the Euler characteristic of a Coxeter group is a simple combinatorial exercise, requiring no more information than the symbol $\Gamma$ and the orders of the finite visible subgroups.

The upshot of all this is that Theorems 7] 10 have direct geometric analogues. Here, for instance, is the one for Theorem 9

Theorem 11. Let $W(\Gamma)$ be a hyperbolic Coxeter group with the symbol $(\dagger)$ of $₫ 3$ where $W(\Psi)$ is an irreducible Weyl group of rank $n$, with exponents $m_{i}$, dimension $d_{\Psi}>1$, Coxeter number $h=2^{p} q$ for $p>0$ and $q$ odd, the first $\ell$ pairs $\left(\Psi, s_{i}\right)$ admissible and the remaining ones specially admissible. Then there is a Galois covering,

$$
M \rightarrow \widehat{M}
$$

of $N$-dimensional hyperbolic manifolds, with deck transformation group $\mathbb{Z} /\left(2^{p}\right)$, and

$$
\operatorname{vol}(\widehat{M})=2^{m n+\ell-p} \prod\left(m_{i}+1\right) \operatorname{covol} W(\Gamma),
$$

where $N \geq n$ is the largest rank of a finite visible subgroup.

We finish with an explicit example. A $(n+1)$-dimensional Lorentzian lattice is an $(n+1)$ dimensional free $\mathbb{Z}$-module equipped with a $\mathbb{Z}$-valued bilinear form of signature $(n, 1)$. For each $n$, there is a unique such, denoted $I_{n, 1}$, that is odd and self-dual (see [25, Theorem V.6], or [20, 21]). By [1], the group $\mathrm{PO}_{n, 1} \mathbb{Z}$ of (projectivised) automorphisms of $I_{n, 1}$ acts properly 
discontinuously by isometries, and with finite covolume, on the hyperbolic space $\mathbb{H}^{n}$ obtained by projectivising the negative norm vectors in $I_{n, 1} \otimes \mathbb{R}$.

Vinberg and Kaplinskaja showed [31,32] that the subgroup $W(\Gamma)$ of $\mathrm{PO}_{n, 1} \mathbb{Z}$ generated by reflections

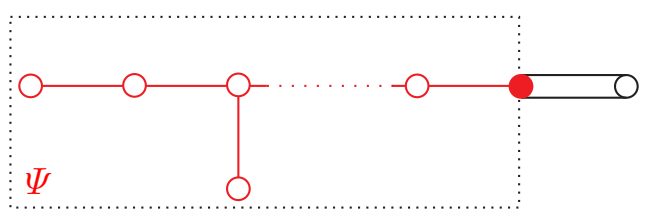
in positive norm vectors has finite index if and only if $n \leq 19$, thus yielding a family of finite covolume $n$-dimensional hyperbolic Coxeter groups for $2 \leq n \leq 19$. Indeed, Conway and Sloane [6, Chapter 28] have shown that for $n \leq 19$ the quotient of $\mathrm{PO}_{n, 1} \mathbb{Z}$ by $W(\Gamma)$ is a subgroup of the automorphism group of the Leech lattice.

The group $W(\Gamma)$ has symbol $\bigcirc-\infty$ when $n=2,3$, and when $4 \leq n \leq 9$, the symbol shown above, with $n+1$ nodes and the fundamental region a non-compact, finite volume $n$-simplex in $\mathbb{H}^{n}$ (for $9<n \leq 19$, the region has a more complicated structure). The $\operatorname{symbol} \Psi$ is,

\begin{tabular}{ccccccc}
\hline$n$ & 4 & 5 & 6 & 7 & 8 & 9 \\
$\Psi$ & $A_{4}$ & $D_{5}$ & $E_{6}$ & $E_{7}$ & $E_{8}$ & $\widetilde{E}_{8}$ \\
\hline
\end{tabular}

Thus, we have $W(\Gamma)$ hyperbolic with the symbol $(\dagger)$ of $\$ 3$ and $W(\Psi)$ an irreducible Weyl group when $3 \leq n \leq 8$ (but for $n=2$ and $9, W(\Psi)$ is the affine Weyl group $\widetilde{A}_{1}$ and $\widetilde{E}_{8}$ ). By Theorems 3 , 4 the pair $(\Psi, s)$, with $s$ the red node, is admissible for $n=4,6$ and 8 .

Thus, let $\Psi=A_{4}, E_{6}$ or $E_{8}$, with $m=\ell=1, h=2^{p} q$ the Coxeter number of these three (with $p=0,2,1$ respectively) and $m_{i}$ the exponents (which are the positive integers relatively prime to the Coxeter number for $A_{4}$ and $E_{8}$, and these, together with 4 and 8 for $E_{6}$ ). The covolume of $W(\Gamma)$ can be computed using the Euler characteristic (5), but in this particular case Siegel [27] gave the volume in terms of a limit which was evaluated in [23] to give the following remarkable formula,

$$
\operatorname{covol} W(\Gamma)=\frac{\left(2^{\frac{n}{2}} \pm 1\right) \pi^{\frac{n}{2}}}{n !} \prod_{k=1}^{\frac{n}{2}}\left|B_{2 k}\right|,
$$

where $B_{2 k}$ is the $2 k$-th Bernoulli number, and we have the minus sign for $n=4,6$ and the plus sign for $n=8$.

A direct application of Theorem 11 and the geometric analogue of Theorem 8 now gives a Galois covering, $M \rightarrow \widehat{M}$, of 4,6 and 8-dimensional hyperbolic manifolds, with deck transformation group $\mathbb{Z} /\left(2^{p}\right)$, and

$$
\operatorname{vol}(\widehat{M})=2^{n-p+1} \frac{\left(2^{\frac{n}{2}} \pm 1\right) \pi^{\frac{n}{2}}}{n !} \prod_{i=1}^{n}\left(m_{i}+1\right) \prod_{k=1}^{\frac{n}{2}}\left|B_{2 k}\right| .
$$

In 4-dimensions this translates to $\frac{8}{3} \pi^{2}$, or a manifold of Euler characteristic $\chi=2$. In 6 dimensions, we do even better if we use instead the geometric analogue of Theorem 10(ii), obtaining the volume $\frac{16}{15} \pi^{3}$, or a manifold of Euler characteristic $\chi=-2$.

\section{References}

[1] Armand Borel and Harish-Chandra, Arithmetic subgroups of algebraic groups, Ann. of Math. (2) 75 (1962), 485-535.

[2] Nicolas Bourbaki, Lie groups and Lie algebras. Chapters 4-6, Elements of Mathematics (Berlin), SpringerVerlag, Berlin, 2002. Translated from the 1968 French original by Andrew Pressley.

[3] Brigitte Brink and Robert B. Howlett, A finiteness property and an automatic structure for Coxeter groups, Math. Ann. 296 (1993), no. 1, 179-190.

[4] R. W. Carter, Conjugacy classes in the Weyl group, Compositio Math. 25 (1972), 1-59.

[5] I. M. Chiswell, The Euler characteristic of graph products and of Coxeter groups, Discrete groups and geometry (Birmingham, 1991), London Math. Soc. Lecture Note Ser., vol. 173, Cambridge Univ. Press, Cambridge, 1992, pp. 36-46. 
[6] J. H. Conway and N. J. A. Sloane, Sphere packings, lattices and groups, 3rd ed., Grundlehren der Mathematischen Wissenschaften [Fundamental Principles of Mathematical Sciences], vol. 290, Springer-Verlag, New York, 1999. With additional contributions by E. Bannai, R. E. Borcherds, J. Leech, S. P. Norton, A. M. Odlyzko, R. A. Parker, L. Queen and B. B. Venkov.

[7] H. S. M. Coxeter, Regular and semi-regular polytopes. III, Math. Z. 200 (1988), no. 1, 3-45.

[8] Vinay V. Deodhar, On the root system of a Coxeter group, Comm. Algebra 10 (1982), no. 6, 611-630.

[9] Brent Everitt, Coxeter groups and hyperbolic manifolds, Math. Ann. 330 (2004), no. 1, 127-150.

[10] - 3-manifolds from Platonic solids, Topology Appl. 138 (2004), no. 1-3, 253-263.

[11] Brent Everitt, John Ratcliffe, and Steven Tschantz, The smallest hyperbolic 6-manifolds, Electron. Res. Announc. Amer. Math. Soc. 11 (2005), 40-46.

[12] Arithmetic hyperbolic 6-manifolds of smallest volume. (in preparation).

[13] Brent Everitt and Colin Maclachlan, Constructing hyperbolic manifolds, Computational and geometric aspects of modern algebra (Edinburgh, 1998), London Math. Soc. Lecture Note Ser., vol. 275, Cambridge Univ. Press, Cambridge, 2000, pp. 78-86.

[14] David Gabai, Robert Meyerhoff, and Peter Milley, Minimum volume cusped hyperbolic 3-manifolds, available at arXiv:0705.4325[math.GT]

[15] Michael Gromov, Volume and bounded cohomology, Inst. Hautes Études Sci. Publ. Math. (1982), no. 56, 5-99 (1983).

[16] Robert B. Howlett, Normalizers of parabolic subgroups of reflection groups, J. London Math. Soc. (2) 21 (1980), no. $1,62-80$.

[17] James E. Humphreys, Reflection groups and Coxeter groups, Cambridge Studies in Advanced Mathematics, vol. 29, Cambridge University Press, Cambridge, 1990.

[18] N. W. Johnson, J. G. Ratcliffe, R. Kellerhals, and S. T. Tschantz, The size of a hyperbolic Coxeter simplex, Transform. Groups 4 (1999), no. 4, 329-353.

[19] Richard Kane, Reflection groups and invariant theory, CMS Books in Mathematics/Ouvrages de Mathématiques de la SMC, 5, Springer-Verlag, New York, 2001.

[20] John Milnor and Dale Husemoller, Symmetric bilinear forms, Springer-Verlag, New York, 1973. Ergebnisse der Mathematik und ihrer Grenzgebiete, Band 73.

[21] A. Neumaier and J. J. Seidel, Discrete hyperbolic geometry, Combinatorica 3 (1983), no. 2, 219-237.

[22] John G. Ratcliffe and Steven T. Tschantz, The volume spectrum of hyperbolic 4-manifolds, Experiment. Math. 9 (2000), no. 1, 101-125.

[23] _ _ Volumes of integral congruence hyperbolic manifolds, J. Reine Angew. Math. 488 (1997), 55-78.

[24] R. W. Richardson, Conjugacy classes of involutions in Coxeter groups, Bull. Austral. Math. Soc. 26 (1982), no. 1, 1-15.

[25] J.-P. Serre, A course in arithmetic, Springer-Verlag, New York, 1973. Translated from the French; Graduate Texts in Mathematics, No. 7.

[26] Carl Ludwig Siegel, Some remarks on discontinuous groups, Ann. of Math. (2) 46 (1945), 708-718.

[27] _ Über die analytische Theorie der quadratischen Formen. II, Ann. of Math. (2) 37 (1936), no. 1, 230263 (German).

[28] Michael Spivak, A comprehensive introduction to differential geometry. Vol. V, 2nd ed., Publish or Perish Inc., Wilmington, Del., 1979. MR 532834 (82g:53003e)

[29] T. A. Springer, A construction of representations of Weyl groups, Invent. Math. 44 (1978), no. 3, $279-293$.

[30] È. B. Vinberg, Hyperbolic groups of reflections, Russian Math. Surveys 40 (1985), no. 1(241), 31-75 (Russian).

[31] - The groups of units of certain quadratic forms, Mat. Sb. (N.S.) 87(129) (1972), 18-36 (Russian).

[32] È. B. Vinberg and I. M. Kaplinskaja, The groups $O_{18,1}(Z)$ and $O_{19,1}(Z)$, Dokl. Akad. Nauk SSSR 238 (1978), no. 6, 1273-1275 (Russian). 